\title{
Asynchronous Effects
}

DANEL AHMAN and MATIJA PRETNAR, University of Ljubljana, Slovenia

\begin{abstract}
We explore asynchronous programming with algebraic effects. We complement their conventional synchronous treatment by showing how to naturally also accommodate asynchrony within them, namely, by decoupling the execution of operation calls into signalling that an operation's implementation needs to be executed, and interrupting a running computation with the operation's result, to which the computation can react by installing interrupt handlers. We formalise these ideas in a small core calculus, called $\lambda_{æ}$. We demonstrate the flexibility of $\lambda_{æ}$ using examples ranging from a multi-party web application, to preemptive multi-threading, to remote function calls, to a parallel variant of runners of algebraic effects. In addition, the paper is accompanied by a formalisation of $\lambda_{æ}$ 's type safety proofs in AGDA, and a prototype implementation of $\lambda_{æ}$ in OCAML.
\end{abstract}

\section{CCS Concepts: • Theory of computation $\rightarrow$ Concurrency; Program constructs; Program semantics.}

Additional Key Words and Phrases: algebraic effects, asynchrony, concurrency, interrupt handling, signals.

\section{ACM Reference Format:}

Danel Ahman and Matija Pretnar. 2021. Asynchronous Effects. Proc. ACM Program. Lang. 5, POPL, Article 24 (January 2021), 28 pages. https://doi.org/10.1145/3434305

\section{INTRODUCTION}

Effectful programming abstractions are at the heart of many modern general-purpose programming languages. They can increase expressiveness by giving access to first-class continuations, but often simply help users to write cleaner code, e.g., by avoiding having to manage a program's memory explicitly in state-passing style, or getting lost in callback hell while programming asynchronously.

An increasing number of language designers and programmers are starting to embrace algebraic effects, where one uses algebraic operations [Plotkin and Power 2002] and effect handlers [Plotkin and Pretnar 2013] to uniformly and user-definably express a wide range of effectful behaviour, ranging from basic examples such as state, rollbacks, exceptions, and nondeterminism [Bauer and Pretnar 2015], to advanced applications in concurrency [Dolan et al. 2018] and statistical probabilistic programming [Bingham et al. 2019], and even quantum computation [Staton 2015].

While covering many examples, the conventional treatment of algebraic effects is synchronous by nature. In it effects are invoked by placing operation calls in one's code, which then propagate outwards until they trigger the actual effect, finally yielding a result to the rest of the computation that has been waiting the whole time. While blocking the computation is indeed sometimes needed, e.g., in the presence of general effect handlers that can execute their continuation any number of times, it forces all uses of algebraic effects to be synchronous, even when this is not necessary, e.g., when the effect involves executing a remote query to which a response is not needed (immediately).

Motivated by the recent interest in the combination of asynchrony and algebraic effects [Dolan et al. 2018; Leijen 2017], we explore what it takes (in terms of language design, safe programming abstractions, and a self-contained core calculus) to accompany the synchronous treatment of

Authors' address: Danel Ahman, danel.ahman@fmf.uni-lj.si; Matija Pretnar, matija.pretnar@fmf.uni-lj.si, University of Ljubljana, Faculty of Mathematics and Physics, Jadranska 21, Ljubljana, SI-1000, Slovenia.

This work is licensed under a Creative Commons Attribution 4.0 International License

(C) 2021 Copyright held by the owner/author(s).

2475-1421/2021/1-ART24

https://doi.org/10.1145/3434305

Proc. ACM Program. Lang., Vol. 5, No. POPL, Article 24. Publication date: January 2021 
algebraic effects with an asynchronous one. At the heart of our approach is the decoupling of the execution of operation calls into signalling that some implementation of an operation needs to be executed, and interrupting a running computation with its result, to which the computation can react by installing interrupt handlers. Importantly, we show that our approach is flexible enough that not all signals need to have a corresponding interrupt, and vice versa, allowing us to also model spontaneous behaviour, such as a user clicking a button or the environment preempting a thread.

While we are not the first ones to work on asynchrony for algebraic effects, the prior work in this area (in the context of general effect handlers) has achieved it by delegating the actual asynchrony to the respective language backends [Dolan et al. 2018; Leijen 2017]. In contrast, in this paper we demonstrate how to capture the combination of asynchrony and algebraic effects in a self-contained core calculus. It is important to emphasise that our aim is not to replace general effect handlers, but instead to complement them with robust primitives tailored to asynchrony-our proposed approach is algebraic by design, so as to be ready for future extensions with general effect handlers.

Paper structure. In Section 2, we give a high-level overview of our approach to asynchrony for algebraic effects. In Section 3 and 4, we distil our ideas into a core calculus, $\lambda_{æ}$, equipped with a small-step semantics, a type-and-effect system, and proofs of type safety. In Section 5, we show $\lambda_{æ}$ in action on examples such as preemptive multi-threading, remote function calls, and a parallel variant of runners of algebraic effects. We conclude, and discuss related and future work in Section 6.

Code. The paper is accompanied by a formalisation of $\lambda_{x}$ 's type safety proofs in AgDA [Ahman 2020], and a prototype implementation of $\lambda_{æ}$ in OCAML, called ÆFF [Pretnar 2020]. For ease of use, we provide them both also as a single virtual machine image [Ahman and Pretnar 2020].

In the AGDA formalisation, we consider only well-typed syntax of a variant of $\lambda_{æ}$ in which the subsumption rule manifests as an explicit coercion, so as to make working with de Bruijn indices less painful. Meanwhile, the ÆFF implementation provides an interpreter and a simple typechecker, but it does not yet support inferring and checking effect annotations. In addition, ÆFF provides a web interface that allows users to enter their programs and interactively click through their executions. ÆFF also comes with implementations of all the examples we present in this paper.

Separately, Poulson [2020] has shown how to implement $\lambda_{æ}$ in Frank [Convent et al. 2020].

\section{ASYNCHRONOUS EFFECTS, BY EXAMPLE}

We begin with a high-level overview of how we accommodate asynchrony within algebraic effects.

\subsection{Conventional Algebraic Effects Are Synchronous by Nature}

We first recall the basic ideas of programming with algebraic effects, illustrating that their traditional treatment is synchronous by nature. For an in-depth overview, we refer to the tutorial by Pretnar [2015], and to the seminal papers of the field [Plotkin and Power 2002; Plotkin and Pretnar 2013].

In this algebraic treatment, sources of computational effects are modelled using signatures of operation symbols op : $A_{\mathrm{op}} \rightarrow B_{\mathrm{op}}$. For instance, one models $S$-valued state using two operations, get : $1 \rightarrow S$ and set : $S \rightarrow 1$; and $E$-valued exceptions using a single operation raise $: E \rightarrow 0$.

Programmers can then invoke the effect that an op $: A_{\mathrm{op}} \rightarrow B_{\mathrm{op}}$ models by placing an operation call op $(V, y . M)$ in their code. Here, the parameter value $V$ has type $A_{\mathrm{op}}$, and the variable $y$, which is bound in the continuation $M$, has type $B_{\text {op }}$. For instance, for set, the parameter $V$ would be the new value of the store, and for get, the variable $y$ would be bound to the current value of the store.

A program written in terms of operation calls is by itself just an inert piece of code. To execute it, programmers have to provide implementations for the operation calls appearing in it. The idea is that an implementation of op $(V, y . M)$ takes $V$ as its input, and its output gets bound to $y$. For instance, this could take the form of defining a suitable effect handler [Plotkin and Pretnar 2013],

Proc. ACM Program. Lang., Vol. 5, No. POPL, Article 24. Publication date: January 2021. 
but could also be given by calls to runners of algebraic effects [Ahman and Bauer 2020], or simply by invoking some (default) top-level (native) implementation. What is important is that some pre-defined piece of code $M_{\mathrm{op}}[V / x]$ gets executed in place of every operation call op $(V, y \cdot M)$.

Now, what makes the conventional treatment of algebraic effects synchronous is that the execution of an operation call op ( $V, y . M)$ blocks until some implementation of op returns a value $W$ to be bound to $y$, so that the execution of the continuation $M[W / y]$ could proceed [Bauer and Pretnar 2014; Kammar et al. 2013]. Conceptually, this kind of blocking behaviour can be illustrated as

$$
\begin{array}{cc}
M_{\mathrm{op}}[V / x] & \sim \text { return } W \\
\uparrow & \downarrow \\
\ldots \sim \text { op }(V, y \cdot M) & M[W / y] \leadsto \ldots
\end{array}
$$

where return $W$ is a computation that causes no effects and simply returns the given value $W$.

While blocking the rest of the computation is needed in the presence of general effect handlers that can execute their continuation any number of times, it forces all uses of algebraic effects to be synchronous, even when this is not necessary, e.g., when the effect in question involves executing a remote query to which a response is not needed immediately, or sometimes never at all.

In the rest of this section, we describe how we decouple the invocation of an operation call from the act of receiving its result, and how we give programmers a means to block execution only when it is necessary. While we end up surrendering some of effect handlers' generality, such as having access to the continuation that captures the rest of the computation to be handled, then in return we get a natural and robust formalism for asynchronous programming with algebraic effects.

\subsection{Outgoing Signals and Incoming Interrupts}

We begin by observing that the execution of an operation call op ( $V, y \cdot M)$, as shown in (1), consists of three distinct phases: (i) signalling that an implementation of op needs to be executed with parameter $V$ (the up-arrow), (ii) executing this implementation (the horizontal arrow), and (iii) interrupting the blocking of $M$ with a value $W$ (the down-arrow). In order to overcome the unwanted side-effects of blocking execution on every operation call, we shall naturally decouple these phases into separate programming concepts, allowing the execution of $M$ to proceed even if (ii) has not yet completed and (iii) taken place. In particular, we decouple an operation call into issuing an outgoing signal, written $\uparrow$ op $(V, M)$, and receiving an incoming interrupt, written $\downarrow$ op $(W, M)$.

It is important to note that while we have used the execution of operation calls to motivate the introduction of signals and interrupts as programming concepts, not all issued signals need to have a corresponding interrupt response, and not all interrupts need to be responses to issued signals, allowing us to also model spontaneous behaviour, such as the environment preempting a thread.

When issuing a signal $\uparrow$ op $(V, M)$, the value $V$ is a payload, such as a location to be looked up or a message to be displayed, aimed at whoever is listening for the given signal. We use the $\uparrow$-notation to indicate that signals issued in sub-computations propagate outwards-in this sense signals behave just like conventional operation calls. However, signals crucially differ from conventional operation calls in that no additional variables are bound in the continuation $M$, making it naturally possible to continue executing $M$ straight after the signal has been issued, e.g., as depicted below:

$$
\begin{aligned}
& \text { op } V \uparrow \\
& \ldots \leadsto \uparrow \mathrm{op}(V, M) \leadsto M \leadsto \ldots
\end{aligned}
$$

As a running example, consider a computation $M_{\text {feedClient }}$, which lets a user scroll through a seemingly infinite feed, e.g., by repeatedly clicking a "next page" button. For efficiency, $M_{\text {feedClient }}$ does not initially cache all the data, but instead requests a new batch of data each time the user is 
nearing the end of the cache. To communicate with the outside world, $M_{\text {feedClient }}$ can issue a signal

$$
\uparrow \text { request (cachedSize }+1, M_{\text {feedClient }} \text { ) }
$$

to request a new batch of data starting from the end of the current cache, or a different signal

$$
\uparrow \text { display ( message, } M_{\text {feedClient }} \text { ) }
$$

to display a message to the user. In both cases, the continuation does not wait for an acknowledgement that the signal was received, but instead continues to provide a seamless experience to the user. It is however worth noting that these signals differ in what $M_{\text {feedClient }}$ expects of them: to the request signal, it expects a response at some future point in its execution, while it does not expect any response to the display signal, illustrating that not every issued signal needs a response.

When the outside world wants to get the attention of a computation, be it in response to a signal or spontaneously, it happens by propagating an interrupt $\downarrow$ op $(W, M)$ to the computation. Here, the value $W$ is again a payload, while $M$ is the computation receiving the interrupt. It is important to note that unlike signals, interrupts are not triggered by the computation itself, but are instead issued by the outside world, and can thus interrupt any sequence of evaluation steps, e.g., as in

$$
\begin{gathered}
\downarrow \text { op } W \\
\ldots \leadsto M \leadsto \downarrow \text { op }(W, M) \leadsto \ldots
\end{gathered}
$$

In our running example, there are two interrupts of interest: $\downarrow$ response (newBatch, $M$ ), which delivers new data to replenish the cache; and $\downarrow$ nextltem $((), M)$, with which the user requests to see the next data item. In both cases, $M$ represents the state of $M_{\text {feedClient }}$ before the interrupt arrived.

We use the $\downarrow$-notation to indicate that interrupts propagate inwards into sub-computations, trying to reach anyone listening for them, and only get discarded when they reach a return. It is worth noting that programmers are not expected to write interrupts explicitly in their programs-instead, interrupts are usually induced by signals issued by other parallel processes, as explained next.

\subsection{A Signal for the Sender Is an Interrupt to the Receiver}

As noted above, the computations we consider do not evolve in isolation, instead they also communicate with the outside world, by issuing outgoing signals and receiving incoming interrupts.

We model the outside world by composing individual computations into parallel processes $P, Q, \ldots$.. To keep the presentation clean and focussed on the asynchronous use of algebraic effects, we consider a very simple model of parallelism: a process is either one of the individual computations being run in parallel, written run $M$, or the parallel composition of two processes, written $P \| Q$.

To capture the signals and interrupts based interaction of processes, our operational semantics includes rules for propagating outgoing signals from individual computations to processes, turning processes' outgoing signals into incoming interrupts for their surrounding world, and propagating incoming interrupts from processes to individual computations. For instance, in our running example, $M_{\text {feedClient }}$ 's request for new data is executed as follows (with the active redexes highlighted):

$$
\begin{aligned}
& \text { run }\left(\uparrow \text { request }\left(V, M_{\text {feedClient }}\right)\right) \| \text { run } M_{\text {feedServer }} \\
\leadsto & \left(\uparrow \text { request }\left(V \text {, run } M_{\text {feedClient }}\right)\right) \| \text { run } M_{\text {feedServer }} \\
\leadsto & \uparrow \text { request }\left(V \text {, run } M_{\text {feedClient }} \| \downarrow \text { request }\left(V \text {, run } M_{\text {feedServer }}\right)\right) \\
\leadsto & \uparrow \text { request }\left(V \text {, run } M_{\text {feedClient }} \| \text { run }\left(\downarrow \text { request }\left(V, M_{\text {feedServer }}\right)\right)\right)
\end{aligned}
$$

Here, the first and the last reduction step respectively propagate signals outwards and interrupts inwards. The middle reduction step corresponds to what we call a broadcast rule-it turns an outward moving signal in one of the processes into an inward moving interrupt for the process parallel to it, while continuing to propagate the signal outwards to any further parallel processes. 


\subsection{Promising to Handle Interrupts}

So far, we have shown that our computations can issue outgoing signals and receive incoming interrupts, and how these evolve when executing parallel processes, but we have not yet said anything about how computations can actually react to incoming interrupts of interest.

In order to react to incoming interrupts, our computations can install interrupt handlers, written

$$
\text { promise (op } x \mapsto M \text { ) as } p \text { in } N
$$

that should be read as: "we promise to handle a future interrupt named op using the computation $M$ in the continuation $N$, with $x$ bound to the payload of the interrupt". Fulfilling this promise consists of executing $M$ and binding its result to the variable $p$ in $N$. This is captured by the reduction rule

$$
\downarrow \text { op }(V \text {, promise }(\text { op } x \mapsto M) \text { as } p \text { in } N) \sim \text { let } p=M[V / x] \text { in } \downarrow \text { op }(V, N)
$$

It is worth noting two things: the interrupt handler is not reinstalled by default, and the interrupt itself keeps propagating inwards into the sub-computation $N$. Regarding the former, programmers can selectively reinstall interrupt handlers when needed, by defining them suitably recursively, e.g., as we demonstrate in Section 2.6. Concerning the latter, then in order to skip certain interrupt handlers for some op, one can carry additional data in op's payload (e.g., a thread ID) and then condition the (non-)triggering of those interrupt handlers on this data, e.g., as we do in Section 5.1.

Interrupts that do not match a given interrupt handler $\left(o p \neq o p^{\prime}\right)$ are simply propagated past it:

$$
\left.\downarrow \mathrm{op}^{\prime}(V \text {, promise }(\text { op } x \mapsto M) \text { as } p \text { in } N) \leadsto \text { promise (op } x \mapsto M\right) \text { as } p \text { in } \downarrow \text { op }^{\prime}(V, N)
$$

Interrupt handlers differ from operation calls in two important aspects. First, they enable user-side post-processing of received data, using $M$, while in operation calls the result is immediately bound in the continuation. Second, and more importantly, their semantics is non-blocking. In particular,

$$
N \leadsto N^{\prime} \quad \text { implies } \quad \text { promise (op } x \mapsto M \text { ) as } p \text { in } N \leadsto \text { promise (op } x \mapsto M \text { ) as } p \text { in } N^{\prime}
$$

meaning that the continuation $N$, and thus the whole computation, can make progress even though no incoming interrupt op has been propagated to the computation from the outside world.

As the observant reader might have noticed, the non-blocking behaviour of interrupt handling means that our operational semantics has to work on open terms because the variable $p$ can appear free in both $N$ and $N^{\prime}$ above. However, it is important to note that $p$ is not an arbitrary variable, but in fact gets assigned a distinguished promise type $\langle X\rangle$ for some value type $X$-we shall crucially

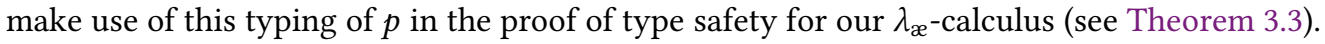

\subsection{Blocking on Interrupts Only When Necessary}

As noted earlier, installing an interrupt handler means making a promise to handle a given interrupt in the future. To check that an interrupt has been received and handled, we provide programmers a means to selectively block execution and await a specific promise to be fulfilled, written await $V$ until $\langle x\rangle$ in $M$, where if $V$ has a promise type $\langle X\rangle$, the variable $x$ bound in $M$ has type $X$. Importantly, the continuation $M$ is executed only when the await is handed a fulfilled promise $\langle V\rangle$ :

$$
\text { await }\langle V\rangle \text { until }\langle x\rangle \text { in } M \leadsto M[V / x]
$$

Revisiting our example of scrolling through a seemingly infinite feed, $M_{\text {feedClient }}$ could use await to block until it has received an initial configuration, such as the batch size used by $M_{\text {feedServer }}$.

As the terminology suggests, this part of $\lambda_{\mathfrak{x}}$ is strongly influenced by existing work on futures and promises [Schwinghammer 2002] for structuring concurrent programs, and their use in modern languages, such as in ScALA [Haller et al. 2020]. While prior work often models promises as writable, single-assignment references, we instead use the substitution of values for ordinary immutable variables (of distinguished promise type) to model that a promise gets fulfilled exactly once. 


\subsection{Putting It All Together}

Finally, we show how to implement our example of scrolling through a seemingly infinite feed. For a simpler exposition, we allow ourselves access to mutable references, though the same can be achieved by rolling one's own state. Further, we use $\uparrow$ op $V$ as a syntactic sugar for $\uparrow$ op ( $V$, return ()).

2.6.1 Client. We implement the client computation $M_{\text {feedClient }}$ as the function client defined below. For presentation purposes, we split the definition of client between multiple code blocks.

First, the client sets up the initial values of the auxiliary references, issues a signal to the server asking for the data batch size that it uses, and then installs a corresponding interrupt handler:

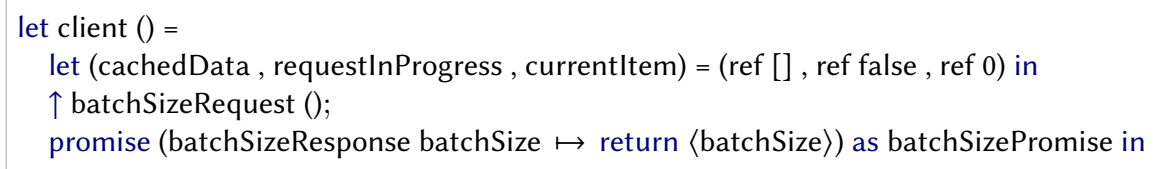

While the server is asynchronously responding to the batch size request, the client sets up an auxiliary function requestNewData, which it later uses to request new data from the server:

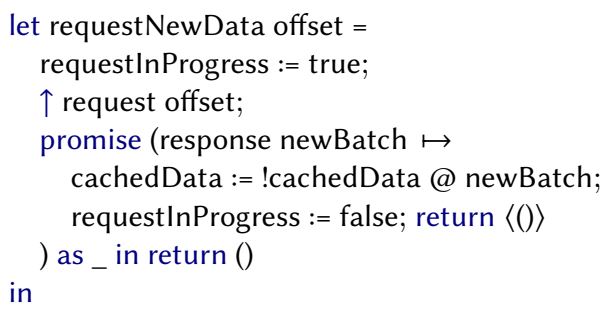

Here, the client first sets a flag indicating that a new data request is in process, then issues a request signal to the server, and finally installs an interrupt handler that updates the cache once the response interrupt arrives. Note that the client does not block while awaiting new data, instead it continues executing, notifying the user to wait and try again once the cache is empty (see below).

Then, the client sets up its main loop, which is a simple recursively defined interrupt handler:

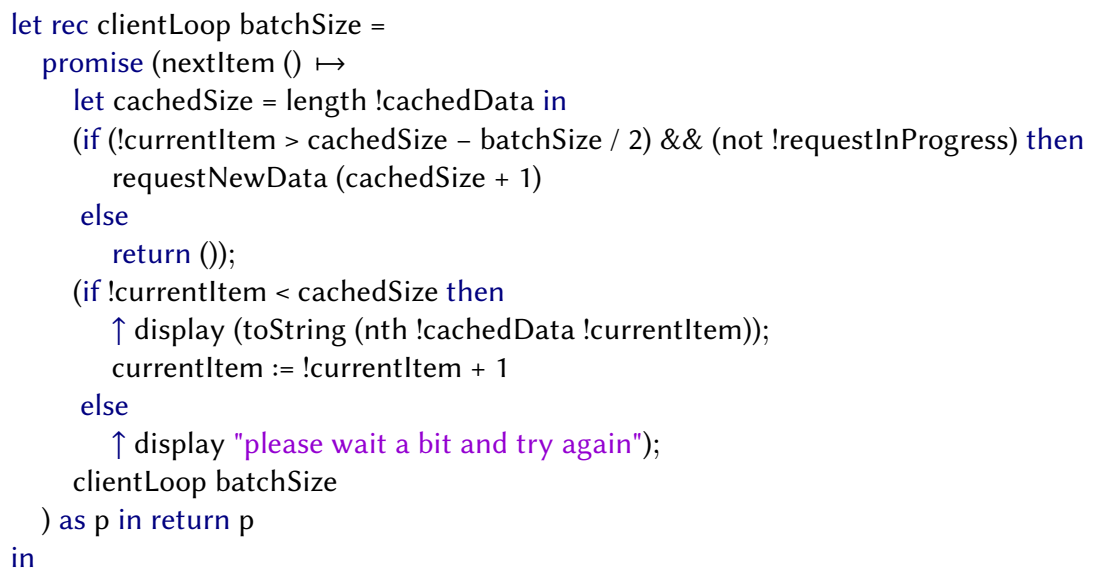

In it, the client listens for a nextltem interrupt from the user to display more data. Once the interrupt arrives, the client checks if its cache is becoming empty-if so, it uses the requestNewData function to request more data from the server. Next, if there is still some data in the cache, the client issues 
a signal to display the next data item to the user. If however the cache is empty, the client issues a signal to display a waiting message to the user. The client then simply recursively reinvokes itself.

As a last step of setting itself up, the client blocks until the server has responded with the batch size it uses, after which the client starts its main loop with the received batch size as follows:

await batchSizePromise until 〈batchSize in clientLoop batchSize

2.6.2 Server. We implement the server computation $M_{\text {feedServer }}$ as the following function:

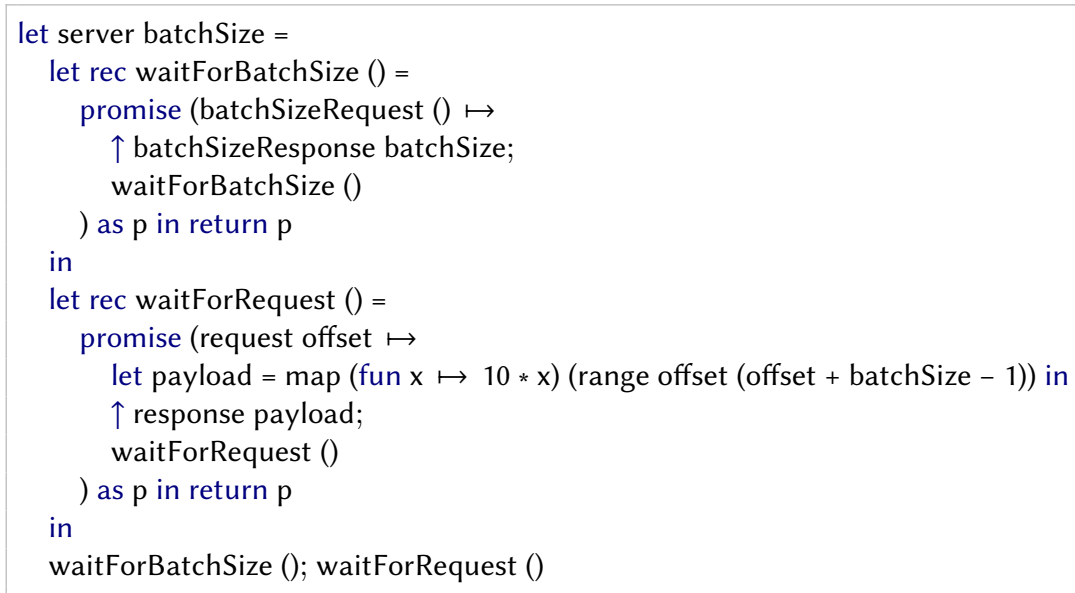

where the computation range $i \mathrm{j}$ returns a list of integers ranging from $\mathrm{i}$ to $\mathrm{j}$ (both inclusive).

The server simply installs two recursively defined interrupt handlers: the first one listens for and responds to client's requests about the batch size it uses; and the second one responds to client's requests for new data. Both interrupt handlers then simply recursively reinstall themselves.

2.6.3 User. We can also simulate the user as a computation. Namely, we implement it as a function that every now and then issues a request to the client to display the next data item:

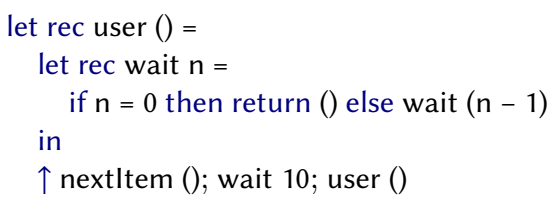

It is straightforward to extend the user also with a handler for display interrupts (we omit it here).

2.6.4 Running the Server, Client, and User in Parallel. Finally, we can simulate our running example in full by running all three computations we defined above as parallel processes, e.g., as follows:

run (server 42) || run (client ()) || run (user ())

\section{A CALCULUS FOR ASYNCHRONOUS EFFECTS: VALUES AND COMPUTATIONS}

We now distil the ideas we introduced in the previous section into a core calculus for programming with asynchronous effects, called $\lambda_{x}$. It is based on Levy et al.'s [2003] fine-grain call-by-value $\lambda$-calculus (FGCBV), and as such, it is a low-level intermediate language to which a corresponding high-level user-facing programming language could be compiled to. In order to better explain the different features of the calculus and its semantics, we split $\lambda_{æ}$ into a sequential part (discussed below) and a parallel part (discussed in Section 4). We note that this separation is purely presentational. 
Values

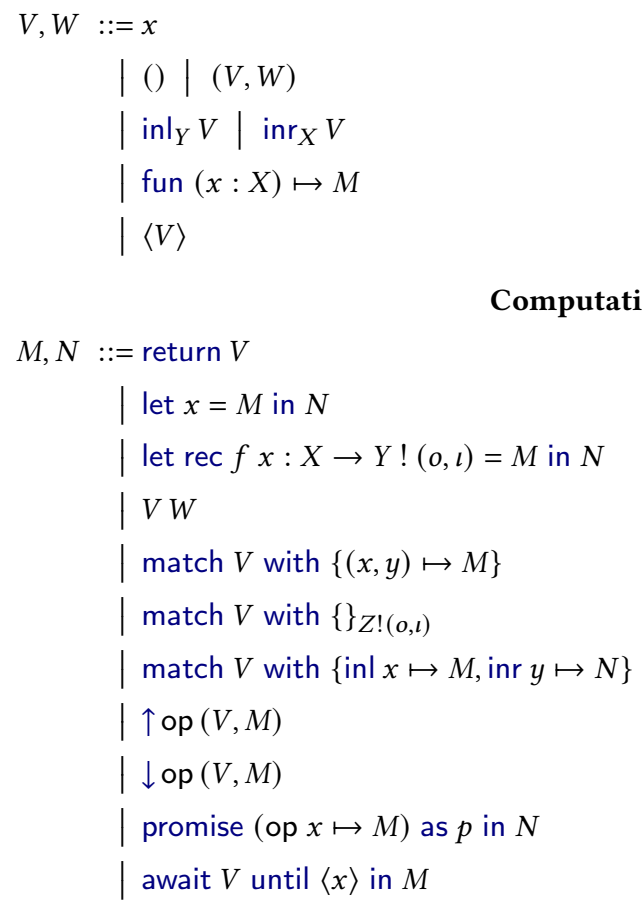

variable

unit and pair

left and right injections

function abstraction

fulfilled promise

\section{Computations}

Fig. 1. Values and computations.

\subsection{Values and Computations}

The syntax of terms is given in Figure 1, stratified into values and computations, as in FGCBV.

Values. The values $V, W, \ldots$ are mostly standard. They include variables, introduction forms for sums and products, and functions. The only $\lambda_{\mathfrak{x}}$-specific value is $\langle V\rangle$, which denotes a fulfilled promise, indicating that the promise of handling some interrupt has been fulfilled with the value $V$.

Computations. The computations $M, N, \ldots$ also include all standard terms from FGCBV: returning values, sequencing, recursion, function application, and elimination forms. Note that we annotate recursive definitions with the type of the function being defined, including the annotations $(o, l)$ that describe the possible effects of the function. While we do not study effect inference in this paper, our experience is that these annotations should make it possible to fully infer types.

The first two computations specific to $\lambda_{æ}$ are signals $\uparrow$ op $(V, M)$ and interrupts $\downarrow$ op $(V, M)$, where the name op is drawn from an assumed set $\Sigma$ of names, $V$ is a data payload, and $M$ is a continuation.

The next $\lambda_{æ}$-specific computation is the interrupt handler promise (op $x \mapsto M$ ) as $p$ in $N$, where $x$ is bound in $M$ and $p$ in $N$. As discussed in the previous section, one should understand this computation as making a promise to handle a future incoming interrupt op by executing the computation $M$. Sub-computations of the continuation $N$ can then explicitly await, when necessary, this promise to be fulfilled by blocking on the promise variable $p$ using the final $\lambda_{x^{-}}$ specific computation term, the awaiting construct await $V$ until $\langle x\rangle$ in $M$. We note that $p$ is an ordinary variable-it just gets assigned the distinguished promise type by the interrupt handler. 


$$
\begin{aligned}
& \text { Standard computation rules } \\
&\text { (fun }(x: X) \mapsto M) V \leadsto M[V / x] \\
& \text { let } x=(\text { return } V) \text { in } N \leadsto N[V / x] \\
& \text { match }(V, W) \text { with }\{(x, y) \mapsto M\} \leadsto M[V / x, W / y] \\
& \text { match }\left(\operatorname{inl}_{Y} V\right) \text { with }\{\operatorname{inl} x \mapsto M, \operatorname{inr} y \mapsto N\} \leadsto M[V / x] \\
& \text { match }\left(\operatorname{inr}_{X} W\right) \text { with }\{\operatorname{inl} x \mapsto M, \operatorname{inr} y \mapsto N\} \leadsto N[W / y] \\
& \text { let rec } f x: X \rightarrow Y !(o, \iota)=M \text { in } N \leadsto N[\text { fun }(x: X) \mapsto \text { let rec } f x: X \rightarrow Y !(o, \iota)=M \text { in } M / f]
\end{aligned}
$$

Algebraicity of signals and interrupt handlers

let $x=(\uparrow$ op $(V, M))$ in $N \leadsto \uparrow$ op $(V$, let $x=M$ in $N)$

let $x=\left(\right.$ promise $($ op $y \mapsto M)$ as $p$ in $N_{1}$ ) in $N_{2} \leadsto$ promise (op $\left.y \mapsto M\right)$ as $p$ in (let $x=N_{1}$ in $N_{2}$ )

\section{Commutativity of signals with interrupt handlers}

$$
\begin{aligned}
& \text { promise (op } x \mapsto M) \text { as } p \text { in } \uparrow \mathrm{op}^{\prime}(V, N) \sim \uparrow \mathrm{op}^{\prime}(V \text {, promise }(\mathrm{op} x \mapsto M) \text { as } p \text { in } N) \\
& \text { Interrupt propagation } \\
& \downarrow \text { op }(V \text {, return } W) \sim \text { return } W \\
& \downarrow \text { op }\left(V, \uparrow \mathrm{op}^{\prime}(W, M)\right) \sim \uparrow \mathrm{op}^{\prime}(W, \downarrow \mathrm{op}(V, M)) \\
& \downarrow \text { op }(V \text {, promise }(\text { op } x \mapsto M) \text { as } p \text { in } N) \leadsto \text { let } p=M[V / x] \text { in } \downarrow \text { op }(V, N) \\
& \downarrow \mathrm{op}^{\prime}(V \text {, promise }(\mathrm{op} x \mapsto M) \text { as } p \text { in } N) \leadsto \text { promise }(\mathrm{op} x \mapsto M) \text { as } p \text { in } \downarrow \mathrm{op}^{\prime}(V, N) \quad\left(\mathrm{op} \neq \mathrm{op}^{\prime}\right)
\end{aligned}
$$

Awaiting a promise to be fulfilled

await $\langle V\rangle$ until $\langle x\rangle$ in $M \sim M[V / x]$

where

\section{Evaluation context rule}

$$
\frac{M \sim N}{\mathcal{E}[M] \sim \mathcal{E}[N]}
$$

$\mathcal{E}::=[] \mid$ let $x=\mathcal{E}$ in $N \mid \uparrow$ op $(V, \mathcal{E}) \mid \downarrow$ op $(V, \mathcal{E}) \mid$ promise $($ op $x \mapsto M)$ as $p$ in $\mathcal{E}$

Fig. 2. Small-step operational semantics of computations.

\subsection{Small-Step Operational Semantics}

We equip $\lambda_{æ}$ with an evaluation contexts based small-step operational semantics, defined using a reduction relation $M \leadsto N$. The reduction rules and evaluation contexts are given in Figure 2.

Computation rules. The first group includes standard reduction rules from FGCBV, such as $\beta$ reducing function applications, sequential composition, and the standard elimination forms. The semantics also includes a rule for unfolding general-recursive definitions. These rules involve standard capture avoiding substitutions $M[V / x]$, defined by straightforward structural recursion.

Algebraicity. This group of reduction rules propagates outwards the signals (resp. interrupt handlers) that have been issued (resp. installed) in sub-computations. While it is not surprising that outgoing signals behave like algebraic operation calls, getting propagated outwards as far as possible, then it is much more curious that the natural operational behaviour of interrupt handlers turns out to be the same. As we shall explain in Section 6, despite using the (systems-inspired) "handler" terminology, mathematically interrupt handlers are in fact a form of algebraic operations. 
Commutativity of signals with interrupt handlers. This rule complements the algebraicity rule for signals, by further propagating them outwards, past any enveloping interrupt handlers. From the perspective of algebraic effects, this rule is an example of two algebraic operations commuting. For this rule to be type safe, the type system ensures that the (promise) variable $p$ cannot appear in $V$.

Interrupt propagation. The handler-operation curiosity does not end with interrupt handlers. This group of reduction rules describes how interrupts are propagated inwards into sub-computations. While $\downarrow$ op $(V, M)$ might look like a conventional operation call, then its operational behaviour instead mirrors that of (deep) effect handling, where one also recursively descends into the computation being handled. The first reduction rule states that we can safely discard an interrupt when it reaches a terminal computation return $W$. The second rule states that we can propagate incoming interrupts past any outward moving signals. The last two rules describe how interrupts interact with interrupt handlers, in particular, that the former behave like effect handling (when understanding interrupt handlers as generalised algebraic operations). On the one hand, if the interrupt matches the interrupt handler it encounters, the corresponding handler code $M$ is executed, and the interrupt is propagated inwards into the continuation $N$. On the other hand, if the interrupt does not match the interrupt handler, it is simply propagated past the interrupt handler into $N$.

Awaiting. The semantics includes a $\beta$-rule for the await construct, allowing the blocked computation $M$ to proceed executing as $M[V / x]$ when await is given a fulfilled promise $\langle V\rangle$.

Evaluation contexts. The semantics allows reductions under evaluation contexts $\mathcal{E}$. Observe that as discussed earlier, the inclusion of interrupt handlers in the evaluation contexts means that reductions involve potentially open terms. Also, differently from the semantics of conventional operation calls [Bauer and Pretnar 2014; Kammar et al. 2013], our evaluation contexts include outgoing signals. As such, the evaluation context rule allows the execution of a computation to proceed even if a signal has not yet been propagated to its receiver, or when an interrupt has not yet arrived. Importantly, the evaluation contexts do not include await, so as to model its intended blocking behaviour. We write $\mathcal{E}[M]$ for the recursive operation of filling the hole [ ] in $\mathcal{E}$ with $M$.

Non-confluence. It is worth noting that the asynchronous design means that the operational semantics is naturally nondeterministic. But more interestingly, the semantics is also not confluent.

For one source of non-confluence, let us consider two reduction sequences of a same (closed) computation, where for better readability, we highlight the active redex for each reduction step:

$$
\downarrow \text { op }\left(V \text {, promise (op } x \mapsto\left(\text { promise }\left(\mathrm{op}^{\prime} y \mapsto M\right) \text { as } q \text { in await } q \text { until }\langle z\rangle \text { in } M^{\prime}\right) \text { ) as } p \text { in } N\right. \text { ) }
$$$$
\leadsto \downarrow \text { op }\left(V \text { , promise } \left(\text { op } x \mapsto\left(\text { promise }\left(\mathrm{op}^{\prime} y \mapsto M\right) \text { as } q \text { in await } q \text { until }\langle z\rangle \text { in } M^{\prime}\right) \text { ) as } p \text { in } N^{\prime}\right.\right. \text { ) }
$$$$
\leadsto \text { let } p=\left(\text { promise }\left(\mathrm{op}^{\prime} y \mapsto M[V / x]\right) \text { as } q \text { in await } q \text { until }\langle z\rangle \text { in } M^{\prime}\right) \text { in } \downarrow \text { op }\left(V, N^{\prime}\right)
$$$$
\leadsto \text { promise (op' } y \mapsto M[V / x]) \text { as } q \text { in await } q \text { until }\langle z\rangle \text { in (let } p=M^{\prime} \text { in } \downarrow \text { op }\left(V, N^{\prime}\right) \text { ) }
$$

and

$\downarrow$ op $\left(V\right.$, promise (op $x \mapsto($ promise (op' $y \mapsto M)$ as $q$ in await $q$ until $\langle z\rangle$ in $\left.M^{\prime}\right)$ ) as $p$ in $N$ )

$$
\begin{aligned}
& \leadsto \text { let } p=\left(\text { promise }\left(\mathrm{op}^{\prime} y \mapsto M[V / x]\right) \text { as } q \text { in await } q \text { until }\langle z\rangle \text { in } M^{\prime}\right) \text { in } \downarrow \text { op }(V, N) \\
& \left.\leadsto \text { promise }\left(\mathrm{op}^{\prime} y \mapsto M[V / x]\right) \text { as } q \text { in await } q \text { until }\langle z\rangle \text { in (let } p=M^{\prime} \text { in } \downarrow \text { op }(V, N)\right)
\end{aligned}
$$

Here, both final computations are temporarily blocked until an incoming interrupt op' is propagated to them and the (promise) variable $q$ gets bound to a fulfilled promise. Until this happens, it is not possible for the blocked continuation $N$ to reduce to $N^{\prime}$ in the latter final computation. 


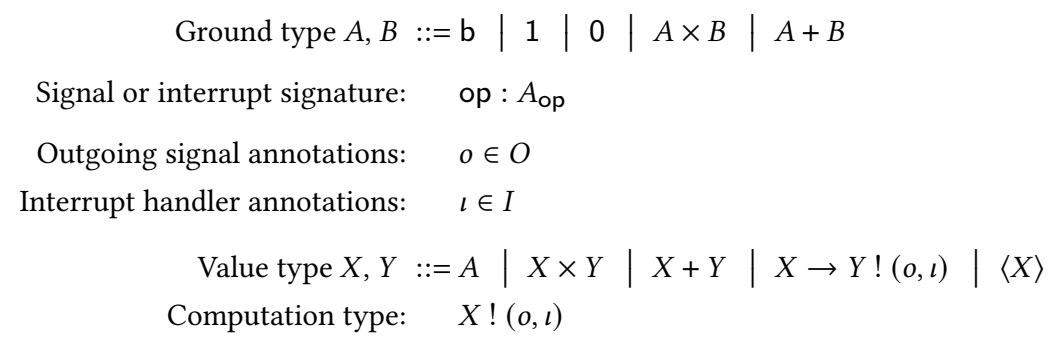

Fig. 3. Value and computation types

Another distinct source of non-confluence concerns the commutativity of outgoing signals with enveloping interrupt handlers. For instance, the following (closed) composite computation

$$
\downarrow \text { op }\left(V \text {, promise (op } x \mapsto \uparrow \mathrm{op}^{\prime}\left(W^{\prime}, M\right)\right) \text { as } p \text { in } \uparrow \mathrm{op}^{\prime \prime}\left(W^{\prime \prime}, N\right) \text { ) }
$$

can nondeterministically reduce to either of the next two computations:

$\uparrow \mathrm{op}^{\prime}\left(W^{\prime}, \uparrow \mathrm{op}^{\prime \prime}\left(W^{\prime \prime}\right.\right.$, let $p=M$ in $\downarrow$ op $\left.\left.(V, N)\right)\right) \quad \uparrow \mathrm{op}^{\prime \prime}\left(W^{\prime \prime}, \uparrow \mathrm{op}^{\prime}\left(W^{\prime}\right.\right.$, let $p=M$ in $\downarrow$ op $\left.\left.(V, N)\right)\right)$

depending on whether we first propagate the interrupt op inwards or the signal op" outwards. As a result, in the resulting two computations, the signals $\mathrm{op}^{\prime}$ and $\mathrm{op}^{\prime \prime}$ get issued in a different order.

\subsection{Type-and-Effect System}

We equip $\lambda_{\Re}$ with a type system in the tradition of type-and-effect systems for algebraic effects and effect handlers [Bauer and Pretnar 2014; Kammar et al. 2013], by extending the simple type system of FGCBV with annotations about possible effects in function and computation types.

3.3.1 Types. We define types in Figure 3, separated into ground, value, and computation types.

As noted in Section 3.1, $\lambda_{\circledast}$ is parameterised over a set $\Sigma$ of signal and interrupt names. To each such name op $\in \Sigma$, we assign a fixed signature op : $A_{\mathrm{op}}$ that specifies the type $A_{\mathrm{op}}$ of the payload of the corresponding signal or interrupt. Crucially, in order to be able to later prove that $\lambda_{æ}$ is type safe (see Theorem 3.3, but also the relevant discussion in Section 6), we restrict these signatures to ground types $A, B, \ldots$, which include standard base, unit, empty, product, and sum types.

Value types $X, Y, \ldots$ extend ground types with function and promise types. The function type $X \rightarrow Y !(o, \iota)$ classifies functions that take $X$-typed arguments to computations classified by the computation type $Y !(o, \iota)$, i.e., ones that return $Y$-typed values, while possibly issuing signals specified by $o$ and handling interrupts specified by $\iota$. The effect annotations $o$ and $\iota$ are drawn from sets $O$ and $I$ whose definitions we discuss below in Section 3.3.2. Finally, the $\lambda_{æ}$-specific promise type $\langle X\rangle$ classifies those promises that can be fulfilled by supplying a value of type $X$.

3.3.2 Effect Annotations. We now explain how we define the sets $O$ and $I$ from which we draw the effect annotations that we use for specifying functions and computations. Traditionally, effect systems for algebraic effects simply use (flat) sets of operation names for effect annotations [Bauer and Pretnar 2014; Kammar et al. 2013]. In $\lambda_{\mathfrak{x}}$, however, we need to be more careful, because triggering an interrupt handler executes a computation that can issue potentially different signals and handle different interrupts from the main program, and we would like to capture this in types.

Signal annotations. First, as outgoing signals do not carry any computational data, we follow the tradition of type-and-effect systems for algebraic effects, and let $O$ be the power $\operatorname{set} \mathcal{P}(\Sigma)$. As such, each $o \in O$ is a subset of the signature $\Sigma$, specifying which signals a computation might issue. 
Interrupt handler annotations. As noted above, for specifying installed interrupt handlers, we cannot use (flat) sets of interrupt names as the effect annotations $\iota \in I$ if we want to track the nested effectful structure. Instead, we define $I$ as the greatest fixed point of a set functor $\Phi$ given by

$$
\Phi(X) \stackrel{\text { def }}{=} \Sigma \Rightarrow(O \times X)_{\perp}
$$

where $\Rightarrow$ is exponentiation, $\times$ is Cartesian product, and $(-)_{\perp}$ is the lifting operation $X_{\perp} \stackrel{\text { def }}{=} X \hookleftarrow\{\perp\}$, and where $\bullet$ is the disjoint union of sets. Formally speaking, $I$ is given by an isomorphism $I \cong \Phi(I)$, but for presentation purposes we leave it implicit and work as if we had a strict equality $I=\Phi(I)$.

Intuitively, each $\iota \in I$ is a possibly infinite nesting of partial mappings of pairs of $O$ - and $I$ annotations to names in $\Sigma$-these pairs of annotations classify the possible effects of the corresponding interrupt handler code. We use the record notation $\iota=\left\{\mathrm{op}_{1} \mapsto\left(o_{1}, \iota_{1}\right), \ldots, \mathrm{op}_{n} \mapsto\left(o_{n}, \iota_{n}\right)\right\}$ to mean that $\iota$ maps the names op ${ }_{1}, \ldots$, op $_{n}$ to the annotations $\left(o_{1}, \iota_{1}\right), \ldots,\left(o_{n}, l_{n}\right)$, and any other names in $\Sigma$ to $\perp$. We write $\iota\left(\mathrm{op}_{i}\right)=\left(o_{i}, l_{i}\right)$ to mean that the annotation $\iota$ maps op $\mathrm{op}_{i}$ to $\left(o_{i}, l_{i}\right)$.

Subtyping and recursive effect annotations. Both $O$ and $I$ come equipped with natural partial orders: for $O, \sqsubseteq_{O}$ is given simply by subset inclusion; and for $I, \sqsubseteq_{I}$ is characterised as follows:

$$
\begin{aligned}
& \iota \sqsubseteq_{I} \iota^{\prime} \text { iff } \quad \forall(\text { op } \in \Sigma)\left(o^{\prime \prime} \in O\right)\left(\iota^{\prime \prime} \in I\right) . \iota(\mathrm{op})=\left(o^{\prime \prime}, \iota^{\prime \prime}\right) \Longrightarrow \\
& \exists\left(o^{\prime \prime \prime} \in O\right)\left(\iota^{\prime \prime \prime} \in I\right) \cdot \iota^{\prime}(\mathrm{op})=\left(o^{\prime \prime \prime}, \iota^{\prime \prime \prime}\right) \wedge o^{\prime \prime} \sqsubseteq_{O} o^{\prime \prime \prime} \wedge \iota^{\prime \prime} \sqsubseteq_{I} \iota^{\prime \prime \prime}
\end{aligned}
$$

We often also use the product order $\emptyset_{O \times I}$, defined as $(o, \iota) \sqsubseteq_{O \times I}\left(o^{\prime}, \iota^{\prime}\right) \stackrel{\text { def }}{=} o \sqsubseteq_{O} o^{\prime} \wedge \iota \sqsubseteq_{I} \iota^{\prime}$. In particular, we use $\sqsubseteq_{O \times I}$ in Section 3.3.3 to define the subtyping relation for $\lambda_{æ}$ 's computation types.

Importantly, the partial orders $\left(O, \sqsubseteq_{O}\right)$ and $\left(I, \sqsubseteq_{I}\right)$ are both $\omega$-complete and pointed, i.e., they are pointed cpos, meaning they have least upper bounds of all increasing $\omega$-chains, and least elements (given by the empty set $\emptyset$ and the constant $\perp$-valued mapping, respectively). As a result, least fixed points of continuous (endo)maps on them are guaranteed to exist. We refer the interested reader to Amadio and Curien [1998] and Gierz et al. [2003] for additional domain-theoretic background.

For $\lambda_{æ}$, we are particularly interested in the least fixed points of continuous maps $f: I \rightarrow I$, so as to specify and typecheck recursive interrupt handler examples, as we illustrate in Section 3.3.4.

We also note that if we were only interested in the type safety of $\lambda_{x}$, and not in typechecking recursively defined interrupt handlers, then we would not need $\left(I, \sqsubseteq_{I}\right)$ to be $\omega$-complete, and could have instead chosen $I$ to be the least fixed point of $\Phi$, which is what we do in our AGDA formalisation. In this case, each interrupt handler annotation $\iota \in I$ would be a finite nesting of partial mappings.

Finally, we envisage that any future full-fledged high-level language based on $\lambda_{\mathfrak{x}}$ would allow users to define their (recursive) effect annotations in a small domain-specific language, providing a syntactic counterpart to the domain-theoretic development we use for typing $\lambda_{æ}$ in this paper.

3.3.3 Typing Rules. We characterise well-typed values using the judgement $\Gamma \vdash V: X$ and welltyped computations using $\Gamma \vdash M: X !(o, \iota)$. In both judgements, $\Gamma$ is a typing context of the form $x_{1}: X_{1}, \ldots, x_{n}: X_{n}$. The rules defining these judgements are respectively given in Figure 4 and 5.

Values. The rules for values are mostly standard. The only $\lambda_{æ}$-specific rule is TyVAL-Promise, which states that in order to fulfil a promise of type $\langle X\rangle$, one has to supply a value of type $X$.

Computations. Analogously to values, the typing rules are standard for the computation terms that $\lambda_{æ}$ inherits from FGCBV, with the $\lambda_{æ}$-rules additionally tracking the effect information $(o, \iota)$.

The $\lambda_{æ}$-specific rule TyComp-Signal states that in order to issue a signal op in a computation with type $X !(o, l)$, we must have op $\in o$ and the type of the payload has to match op's signature.

The rule TyСомр-Promise states that the interrupt handler code $M$ has to return a fulfilled promise of type $\langle X\rangle$, for some type $X$, while possibly issuing signals $o^{\prime}$ and handling interrupts $\iota^{\prime}$, 


\begin{tabular}{|c|c|c|c|c|}
\hline TYVAL-VAR & TrVAL-Unit & $\begin{array}{l}\text { TYVAL-PAIR } \\
\Gamma \vdash V: X\end{array}$ & $\Gamma \vdash W: Y$ & $\begin{array}{l}\text { TyVal-Promise } \\
\Gamma \vdash V: X\end{array}$ \\
\hline$\overline{\Gamma, x: X, \Gamma^{\prime} \vdash x: X}$ & $\overline{\Gamma \vdash(): 1}$ & $\Gamma \vdash(V, W)$ & $: X \times Y$ & $\overline{\Gamma \vdash\langle V\rangle:\langle X\rangle}$ \\
\hline $\begin{array}{l}\text { TyVAL-InL } \\
\qquad \Gamma \vdash V: X\end{array}$ & $\begin{array}{l}\text { TyVAL-INR } \\
\qquad \Gamma \vdash W: Y\end{array}$ & \multicolumn{3}{|c|}{$\begin{array}{l}\text { TrVAL-FUn } \\
\qquad \Gamma, x: X \vdash M: Y !(o, \iota)\end{array}$} \\
\hline$\Gamma \vdash \operatorname{inl}_{Y} V: X+Y$ & $\overline{\Gamma+\operatorname{inr}_{X} W: 2}$ & \multicolumn{3}{|c|}{$\overline{\Gamma \vdash \text { fun }(x: X) \mapsto M: X \rightarrow Y !(o, \iota)}$} \\
\hline
\end{tabular}

Fig. 4. Value typing rules.

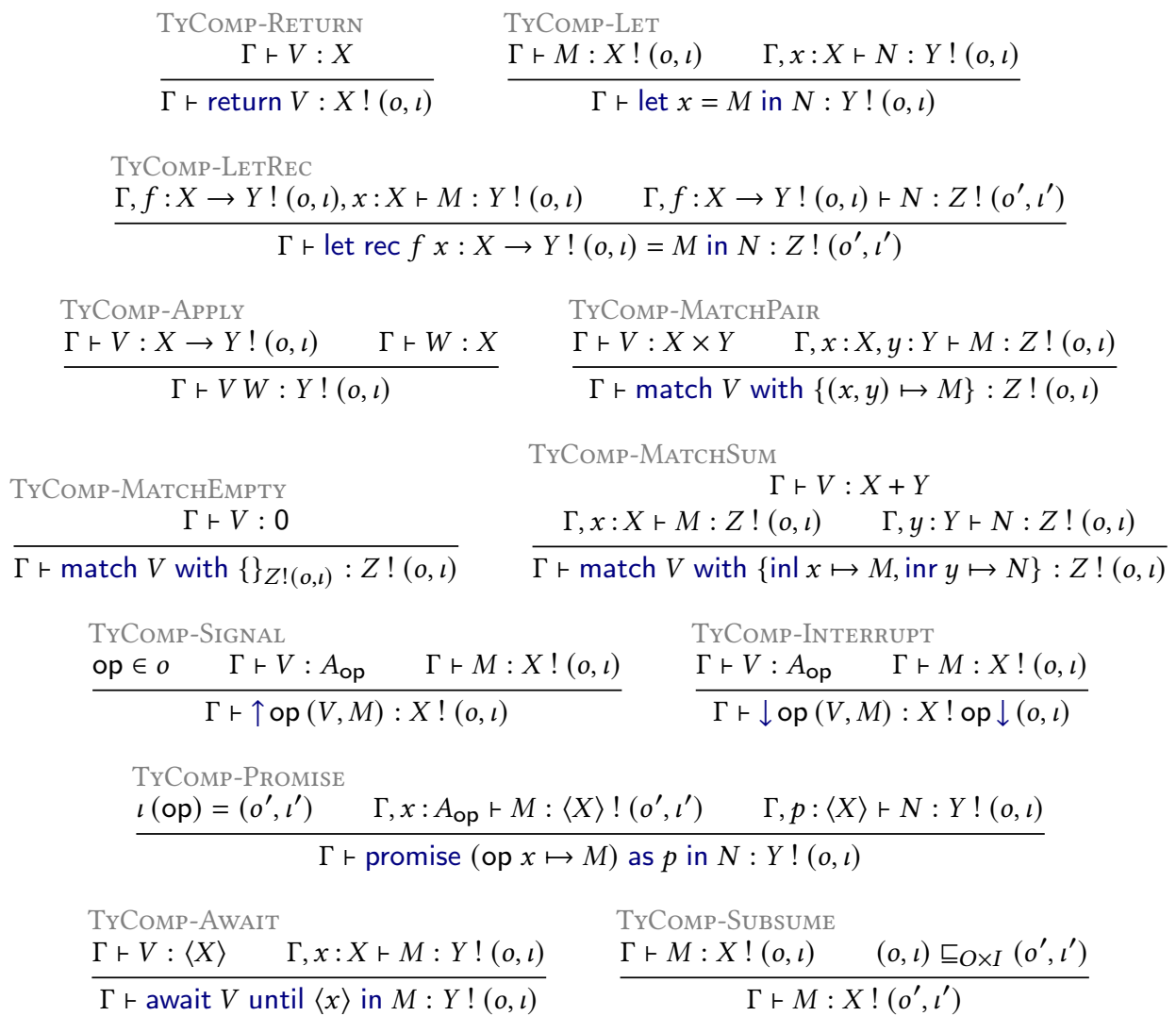

Fig. 5. Computation typing rules.

both of which are determined by the effect annotation $\iota$ of the entire computation, i.e., $\iota$ op $)=\left(o^{\prime}, \iota^{\prime}\right)$. The variable $p$ bound in the continuation, which sub-computations can block on to await a given interrupt to arrive and be handled, also gets assigned the promise type $\langle X\rangle$. It is worth noting that one could have had $M$ simply return values of type $X$, but at the cost of not being able to implement some of the more interesting examples, e.g., guarded interrupt handlers in Section 5.1. At the same time, for $\lambda_{æ}$ 's type safety, it is crucial that $p$ would have remained assigned the promise type $\langle X\rangle$.

The rule TyComp-AwAIT simply says that when awaiting a promise of type $\langle X\rangle$ to be fulfilled, the continuation $M$ can refer to the promised value (in the future) using the variable $x$ of type $X$. 
The rule TyComp-InTERRUPT is used to type incoming interrupts. In particular, when the outside world propagates an interrupt op to a computation $M$ of type $X !(o, l)$, the resulting computation $\downarrow$ op $(V, M)$ gets assigned the type $X$ ! op $\downarrow(o, l)$, where the interrupt op also acts on the effect annotations. Intuitively, op $\downarrow(o, l)$ mimics the act of triggering interrupt handlers for op at the level of effect annotations. Formally, we define this action of interrupts on effect annotations as follows:

$$
\text { op } \downarrow(o, \iota) \stackrel{\text { def }}{=} \begin{cases}\left(o \cup o^{\prime}, \iota[\mathrm{op} \mapsto \perp] \cup \iota^{\prime}\right) & \text { if } \iota(\mathrm{op})=\left(o^{\prime}, \iota^{\prime}\right) \\ (o, \iota) & \text { otherwise }\end{cases}
$$

In other words, if $M$ has any interrupt handlers installed for op, then $\iota$ (op) $=\left(o^{\prime}, \iota^{\prime}\right)$, where $\left(o^{\prime}, \iota^{\prime}\right)$ specifies the effects of said interrupt handler code. Now, when the inward propagating interrupt op reaches those interrupt handlers, it triggers the execution of the corresponding handler code, and thus the entire computation $\downarrow$ op $(V, M)$ can also issue signals in $o^{\prime}$ and handle interrupts in $\iota^{\prime}$.

The notation $\iota$ op $\mapsto \perp$ ] sets $\iota$ to $\perp$ at op, and leaves it unchanged elsewhere. In particular, mapping op to $\perp$ captures that the interrupt triggers all corresponding interrupt handlers in $M$.

The join-semilattice structure $o \cup o^{\prime} \in O$ is given by the union of sets, while $\iota \cup \iota^{\prime} \in I$ is given by

$$
\iota \cup \iota^{\prime} \stackrel{\text { def }}{=} \lambda \mathrm{op} . \begin{cases}\left(o^{\prime \prime} \cup o^{\prime \prime \prime}, \iota^{\prime \prime} \cup \iota^{\prime \prime \prime}\right) & \text { if } \iota \text { (op })=\left(o^{\prime \prime}, \iota^{\prime \prime}\right) \wedge \iota^{\prime}(\mathrm{op})=\left(o^{\prime \prime \prime}, \iota^{\prime \prime \prime}\right) \\ \left(o^{\prime \prime}, \iota^{\prime \prime}\right) & \text { if } \iota \text { (op })=\left(o^{\prime \prime}, \iota^{\prime \prime}\right) \wedge \iota^{\prime}(\mathrm{op})=\perp \\ \left(o^{\prime \prime \prime}, \iota^{\prime \prime \prime}\right) & \text { if } \iota(\mathrm{op})=\perp \wedge \iota^{\prime}(\mathrm{op})=\left(o^{\prime \prime \prime}, \iota^{\prime \prime \prime}\right) \\ \perp & \text { if } \iota(\mathrm{op})=\perp \wedge \iota^{\prime}(\mathrm{op})=\perp\end{cases}
$$

We also note that the action op $\downarrow$ (-) has various useful properties, which we use in later proofs (where we write $\pi_{1}$ and $\pi_{2}$ for the two projections associated with the Cartesian product $O \times I$ ):

LEMMA 3.1.

(1) $o \sqsubseteq O \pi_{1}(\mathrm{op} \downarrow(o, \iota))$.

(2) If $\iota$ (op) $=\left(o^{\prime}, \iota^{\prime}\right)$, then $\left(o^{\prime}, \iota^{\prime}\right) \sqsubseteq O \times I$ op $\downarrow(o, l)$.

(3) If op $\neq \mathrm{op}^{\prime}$ and $\iota\left(\mathrm{op}^{\prime}\right)=\left(o^{\prime}, \iota^{\prime}\right)$, then $\left(o^{\prime}, \iota^{\prime}\right) \sqsubseteq_{O \times I}\left(\pi_{2}(\mathrm{op} \downarrow(o, \iota))\right)\left(\mathrm{op}^{\prime}\right)$.

Finally, the rule TyComp-SuBsume allows subtyping. To simplify the presentation, we consider a limited form of subtyping, in which we shallowly relate only signal and interrupt annotations.

3.3.4 Typechecking Recursively Defined Interrupt Handlers. We conclude discussing $\lambda_{\mathfrak{x}}$ 's typeand-effect system by briefly returning to the reason why we defined our effect annotations using lightweight domain theory in the first place, namely, so as to typecheck recursive interrupt handlers.

As an example, we recall the following fragment of the server code from Section 2.6.2:

let rec waitForBatchSize ()$=$

promise (batchSizeReq ()$\mapsto \uparrow$ batchSizeResp batchSize; waitForBatchSize ()$)$ as $p$ in return $p$

Here, waitForBatchSize () is an interrupt handler for batchSizeReq that recursively reinstalls itself immediately after issuing a batchSizeResp signal. Due to its recursive definition, it is not surprising that the type of waitForBatchSize should also be given recursively, in particular, if we want to give it a more precise type than one which simply says that any effect is possible.

To this end, we assign waitForBatchSize the type $1 \rightarrow\langle 1\rangle !\left(\emptyset, \iota_{\mathrm{b}}\right)$, where $\iota_{\mathrm{b}}$ is the least fixed point of the continuous map $\iota \mapsto\{$ batchSizeReq $\mapsto(\{$ batchSizeResp $\}, \iota)\}: I \rightarrow I$, i.e.,

$$
\iota_{\mathrm{b}}=\{\text { batchSizeReq } \mapsto(\{\text { batchSizeResp }\},\{\text { batchSizeReq } \mapsto(\{\text { batchSizeResp }\}, \ldots)\})\}
$$

As such, $\left(\emptyset, \iota_{\mathrm{b}}\right)$ captures that at the top level waitForBatchSize () installs an interrupt handler and issues no signals, and that every batchSizeReq interrupt causes a signal to be issued and the interrupt handler to be reinstalled. Checking that waitForBatchSize has the type $1 \rightarrow\langle 1\rangle !\left(\emptyset, \iota_{\mathrm{b}}\right)$ 
involves unfolding the definition of $\iota_{\mathrm{b}}$ and using subtyping. The latter is needed when we recursively call waitForBatchSize () where a computation of type $\langle 1\rangle$ ! (\{batchSizeResp $\left.\}, \iota_{\mathrm{b}}\right)$ is expected.

\subsection{Type Safety}

We now prove type safety for the sequential part of $\lambda_{æ}$, showing that "well-typed programs do not go wrong”. As usual, we split type safety into progress and preservation [Wright and Felleisen 1994].

3.4.1 Progress. The progress result says that well-typed closed computations can either make a step of reduction, or are already in a well-defined result form (and thus have stopped reducing).

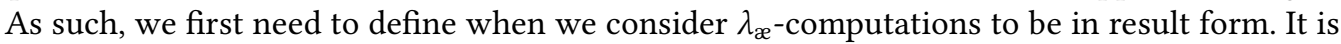
important to note that for $\lambda_{æ}$, the result forms have to also incorporate the temporary blocking while computations await some promise (variable) $p$ to be fulfilled. Therefore, as a first step, we characterise such computations using the judgement $p \& M$, given by the following three rules:

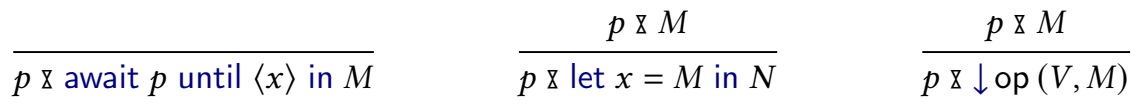

Next, we characterise $\lambda_{æ}$ 's result forms using the judgements CompRes $\langle\Psi \mid M\rangle$ and $\operatorname{Run} \operatorname{Res}\langle\Psi \mid M\rangle$ :

$$
\begin{gathered}
\frac{\operatorname{CompRes}\langle\Psi \mid M\rangle}{\operatorname{CompRes}\langle\Psi| \uparrow \text { op }(V, M)\rangle} \quad \frac{\operatorname{RunRes}\langle\Psi \mid M\rangle}{\operatorname{CompRes}\langle\Psi \mid M\rangle} \\
\frac{\operatorname{RunRes}\langle\Psi \cup\{p\} \mid N\rangle}{\operatorname{RunRes}\langle\Psi| \text { return } V\rangle} \quad \frac{p \in \Psi \quad p \text { \& } M}{\operatorname{RunRes}\langle\Psi| \operatorname{promise}(\text { op } x \mapsto M) \text { as } p \text { in } N\rangle} \quad \frac{p \operatorname{RunRes}\langle\Psi \mid M\rangle}{\operatorname{Rin}}
\end{gathered}
$$

In these judgements, $\Psi$ is a set of (promise) variables that have been bound by interrupt handlers enveloping the computation. These judgements express that a computation $M$ is in a (top-level) result form CompRes $\langle\Psi \mid M\rangle$ when, considered as a tree, it has a shape in which all signals are towards the root, interrupt handlers are in the intermediate nodes, and the leaves contain return values and computations that are temporarily blocked while awaiting one of the promise variables in $\Psi$ to be fulfilled. The slightly mysterious name of the intermediate judgement $\operatorname{RunRes}\langle\Psi \mid M\rangle$ will become clear in Section 4.4. The finality of these result forms is captured by the next lemma.

Lemma 3.2. Given $\Psi$ and $M$ such that $\operatorname{CompRes}\langle\Psi \mid M\rangle$, then there exists no $N$ with $M \leadsto N$.

We are now ready to state and prove the progress theorem for the sequential part of $\lambda_{\mathfrak{x}}$.

Theorem 3.3. Given a well-typed computation $\Gamma \vdash M: Y !(o, \iota)$, where $\Gamma=x_{1}:\left\langle X_{1}\right\rangle, \ldots, x_{n}:\left\langle X_{n}\right\rangle$, then either $(i)$ there exists an $N$ such that $M \leadsto N$, or (ii) we have CompRes $\left\langle\left\{x_{1}, \ldots, x_{n}\right\} \mid M\right\rangle$.

Proof. The proof is standard and proceeds by induction on the derivation of $\Gamma \vdash M: Y !(o, \iota)$. For instance, if the derivation ends with a typing rule for function application or pattern-matching, we use an auxiliary canonical forms lemma to show that the value involved is either a function abstraction or in constructor form-thus $M$ can $\beta$-reduce and we prove (i). Here we crucially rely on the context $\Gamma$ having the specific assumed form $x_{1}:\left\langle X_{1}\right\rangle, \ldots, x_{n}:\left\langle X_{n}\right\rangle$. If the derivation ends with TyComp-Await, then we use a canonical forms lemma to show that the promise value is either a variable in $\Gamma$, in which case we prove (ii), or in constructor form, in which case we prove (i). If the derivation however ends with a typing rule for any of the terms figuring in the evaluation contexts $\mathcal{E}$, then we proceed based on using the induction hypothesis on the corresponding continuation.

Corollary 3.4. Given a well-typed closed computation $\vdash M: X !(o, \iota)$, then either $(i)$ there exists a computation $N$ such that $M \leadsto N$, or (ii) $M$ is already in result form, i.e., we have CompRes $\langle\emptyset \mid M\rangle$. 
3.4.2 Type Preservation. The type preservation result says that reduction preserves well-typedness.

The results that we present in this section use standard substitution lemmas. For instance, given $\Gamma, x: X, \Gamma^{\prime} \vdash M: Y !(o, \iota)$ and $\Gamma \vdash V: X$, then we can show that $\Gamma, \Gamma^{\prime} \vdash M[V / x]: Y !(o, \iota)$. In the following we also use standard typing inversion lemmas. For example, given $\Gamma \vdash \downarrow$ op $(V, M): X !(o, \iota)$, then we can show that $\Gamma \vdash V: A_{\mathrm{op}}$ and $\Gamma \vdash M: X !$ op $\downarrow\left(o^{\prime}, \iota^{\prime}\right)$, such that op $\downarrow\left(o^{\prime}, \iota^{\prime}\right) \sqsubseteq O \times I(o, \iota)$.

As the proof of type preservation proceeds by induction on reduction steps, we find it useful to define an auxiliary typing judgement for evaluation contexts, written $\Gamma \vdash\left[\Gamma^{\prime} \mid X !(o, \iota)\right] \mathcal{E}: Y !\left(o^{\prime}, \iota^{\prime}\right)$, which we then use to prove the evaluation context rule case of the proof. Here, $\Gamma^{\prime}$ is the context of variables bound by the interrupt handlers in $\mathcal{E}$, and $X !(o, \iota)$ is the type of the hole [ ]. This judgement is given using rules similar to those for computations, including subtyping, e.g., we have

$$
\frac{\iota^{\prime}(\mathrm{op})=\left(o^{\prime \prime}, \iota^{\prime \prime}\right) \quad \Gamma, x: A_{\mathrm{op}} \vdash M:\langle Y\rangle !\left(o^{\prime \prime}, \iota^{\prime \prime}\right) \quad \Gamma, p:\langle Y\rangle \vdash\left[\Gamma^{\prime} \mid X !(o, \iota)\right] \mathcal{E}: Z !\left(o^{\prime}, \iota^{\prime}\right)}{\Gamma \vdash\left[p:\langle Y\rangle, \Gamma^{\prime} \mid X !(o, \iota)\right] \text { promise }(\text { op } x \mapsto M) \text { as } p \text { in } \mathcal{E}: Z !\left(o^{\prime}, \iota^{\prime}\right)}
$$

It is thus straightforward to relate this typing of evaluation contexts with that of computations.

LEMMA 3.5.

$\Gamma \vdash \mathcal{E}[M]: Y !\left(o^{\prime}, \iota^{\prime}\right) \Leftrightarrow \exists \Gamma^{\prime}, X, o, \iota . \Gamma \vdash\left[\Gamma^{\prime} \mid X !(o, \iota)\right] \mathcal{E}: Y !\left(o^{\prime}, \iota^{\prime}\right) \wedge \Gamma, \Gamma^{\prime} \vdash M: X !(o, \iota)$.

We are now ready to state and prove the type preservation theorem for the sequential part of $\lambda_{æ}$.

Theorem 3.6. Given $\Gamma \vdash M: X !(o, \iota)$ and $M \leadsto N$, then we have $\Gamma \vdash N: X !(o, \iota)$.

Proof. The proof is standard and proceeds by induction on the derivation of $M \leadsto N$, using typing inversion lemmas depending on the structure forced upon $M$ by the last rule used in $M \leadsto N$.

There are four cases of interest in this proof. The first two concern the interaction of incoming interrupts and interrupt handlers. On the one hand, if the given derivation of $\sim$ ends with

$$
\downarrow \text { op }(V \text {, promise }(\text { op } x \mapsto M) \text { as } p \text { in } N) \sim \text { let } p=M[V / x] \text { in } \downarrow \text { op }(V, N)
$$

then in order to type the right-hand side of this rule, we are led to use subtyping with Lemma 3.1 (2), so as to show that M's effect information is included in op $\downarrow(o, \iota)$. On the other hand, given

$\downarrow \mathrm{op}^{\prime}(V$, promise $(\mathrm{op} x \mapsto M)$ as $p$ in $N) \leadsto$ promise (op $\left.x \mapsto M\right)$ as $p$ in $\downarrow \mathrm{op}^{\prime}(V, N) \quad\left(\mathrm{op} \neq \mathrm{op}^{\prime}\right)$

then in order to type the right-hand side of this rule, we are led to use subtyping with Lemma 3.1 (3), so as to show that after acting on $(o, \iota)$ with op', op remains mapped to $M$ 's effect information.

The third case of interest concerns the commutativity of signals with interrupt handlers:

$$
\text { promise (op } x \mapsto M) \text { as } p \text { in } \uparrow \mathrm{op}^{\prime}(V, N) \sim \uparrow \mathrm{op}^{\prime}(V \text {, promise (op } x \mapsto M) \text { as } p \text { in } N \text { ) }
$$

where in order to type the signal's payload $V$ in the right-hand side, it is crucial that the promisetyped variable $p$ cannot appear in $V$-this is ensured by our type system that restricts the signatures op : $A_{\mathrm{op}}$ to ground types. As a result, we can strengthen the typing context of $V$ by removing $p$.

Finally, in the evaluation context rule case, we use the induction hypothesis with Lemma 3.5.

Interestingly, the proof of Theorem 3.6 tells us that if one were to consider a variant of $\lambda_{\mathfrak{x}}$ in

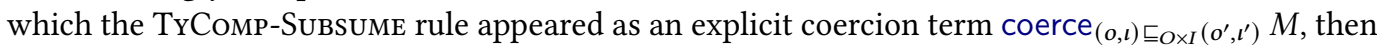
the right-hand sides of the two interrupt propagation rules highlighted in the above proof would also need to involve such coercions, corresponding to the uses of Lemma 3.1. This however means that other computations involved in these reduction rules would also need to be type-annotated.

\section{A CALCULUS FOR ASYNCHRONOUS EFFECTS: PARALLEL PROCESSES}

Next, we describe the parallel part of $\lambda_{æ}$. Similarly to the sequential part, we again present the corresponding syntax, a small-step semantics, a type-and-effect system, and type safety results. 


\section{Individual computations}

$\frac{M \sim N}{\operatorname{run} M \leadsto \operatorname{run} N}$

Signal hoisting

$\operatorname{run}(\uparrow$ op $(V, M)) \sim \uparrow$ op $(V$, run $M)$

Broadcasting

$\uparrow$ op $(V, P) \| Q \sim \uparrow$ op $(V, P \| \downarrow$ op $(V, Q))$

$P \| \uparrow$ op $(V, Q) \sim \uparrow$ op $(V, \downarrow$ op $(V, P) \| Q)$

\section{Interrupt propagation}

$$
\begin{aligned}
\downarrow \text { op }(V, \operatorname{run} M) & \sim \operatorname{run}(\downarrow \text { op }(V, M)) \\
\downarrow \text { op }(V, P \| Q) & \sim \downarrow \text { op }(V, P) \| \downarrow \text { op }(V, Q) \\
\downarrow \text { op }\left(V, \uparrow \mathrm{op}^{\prime}(W, P)\right) & \sim \uparrow \mathrm{op}^{\prime}(W, \downarrow \text { op }(V, P))
\end{aligned}
$$

\section{Evaluation context rule}

$$
\frac{P \sim Q}{\mathcal{F}[P] \sim \mathcal{F}[Q]}
$$

$$
\begin{gathered}
\text { where } \\
\mathcal{F}::=[]|\mathcal{F}\|Q|P \| \mathcal{F}| \uparrow \mathrm{op}(V, \mathcal{F}) \mid \downarrow \text { op }(V, \mathcal{F})
\end{gathered}
$$

Fig. 6. Small-step operational semantics of parallel processes.

\subsection{Parallel Processes}

To keep the presentation focussed on the asynchronous use of algebraic effects, we consider a very simple model of parallelism: a process is either an individual computation or the parallel composition of two processes. To facilitate interactions between processes, they also contain outward propagating signals and inward propagating interrupts. Formally, the syntax of parallel processes is

$$
P, Q::=\operatorname{run} M|P \| Q| \uparrow \text { op }(V, P) \mid \downarrow \text { op }(V, P)
$$

Note that processes do not include interrupt handlers-these are local to individual computations.

We leave first-class processes and their dynamic creation for future work, as discussed in Section 6.

\subsection{Small-Step Operational Semantics}

We equip the parallel part of $\lambda_{æ}$ with a small-step semantics that naturally extends that of $\lambda_{æ}$ 's sequential part. The semantics is defined using a reduction relation $P \leadsto Q$, as given in Figure 6 .

Individual computations. This reduction rule states that, as processes, individual computations evolve according to the small-step operational semantics $M \leadsto N$ we defined for them in Section 3.2.

Signal hoisting. This rule propagates signals out of individual computations. It is important to note that we only hoist those signals that have propagated to the outer boundary of a computation.

Broadcasting. The broadcast rules turn outward moving signals in one process into inward moving interrupts for the process parallel to it, while continuing to propagate the signals outwards to any further parallel processes. The latter ensures that the semantics is compositional.

Interrupt propagation. These three rules simply propagate interrupts inwards into individual computations, into all branches of parallel compositions, and past any outward moving signals.

Evaluation contexts. Analogously to the semantics of computations, the semantics of processes also includes a context rule, which allows reductions under evaluation contexts $\mathcal{F}$. Observe that compared to the evaluation contexts for computations, those for processes do not bind variables. 


$\begin{array}{llll}\text { TyProc-Run } & \text { TyProc-Par } & \begin{array}{c}\text { TyProc-Signal } \\ \text { op } \in \text { signals-of }(C)\end{array} & \\ \frac{\Gamma \vdash M: X !(o, \iota)}{\Gamma \vdash \operatorname{run} M: X ! !(o, \iota)} & \frac{\Gamma \vdash P: C}{\Gamma \vdash P\|Q: C\| D} & \frac{\Gamma \vdash V: A_{\mathrm{op}} \quad \Gamma \vdash P: C}{\Gamma \vdash \uparrow \mathrm{op}(V, P): C} & \frac{\Gamma \vdash V: A_{\mathrm{op}} \quad \Gamma \vdash P: C}{\Gamma \vdash \downarrow \text { op }(V, P): \mathrm{op} \downarrow C}\end{array}$

Fig. 7. Process typing rules.

\subsection{Type-and-Effect System}

Analogously to its sequential part, we also equip $\lambda_{æ}$ 's parallel part with a type-and-effect system.

Types. The types of processes are designed to match their parallel structure-they are given by

$$
C, D::=X ! !(o, \iota) \mid C \| D
$$

Intuitively, $X ! !(o, \iota)$ is a process type of an individual computation of type $X !(o, \iota)$, and $C \| D$ is the type of the parallel composition of two processes that respectively have types $C$ and $D$.

Typing judgements. Well-typed processes are characterised using the judgement $\Gamma \vdash P: C$. We present the typing rules in Figure 7 . While our processes are not currently higher-order, we allow non-empty contexts $\Gamma$ to model the possibility of using libraries and top-level function definitions.

The rules TyProc-Run and TyProc-Par capture the earlier intuition about the types of processes matching their parallel structure. The rules TyProc-SignAL and TyProc-INTERruPT are similar to the corresponding rules from Figure 5. The signal annotations of a process type are calculated as

$$
\text { signals-of }(X ! !(o, \iota)) \stackrel{\text { def }}{=} o \quad \text { signals-of }(C \| D) \stackrel{\text { def }}{=} \text { signals-of }(C) \cup \text { signals-of }(D)
$$

and the action of interrupts on process types op $\downarrow C$ extends the action on effect annotations as

$$
\text { op } \downarrow(X ! !(o, l)) \stackrel{\text { def }}{=} X ! !(\text { op } \downarrow(o, l)) \quad \text { op } \downarrow(C \| D) \stackrel{\text { def }}{=}(\text { op } \downarrow C) \|(\text { op } \downarrow D)
$$

by propagating the interrupt towards the types of individual computations. We then have:

Lemma 4.1. For any process type $C$ and interrupt op, we have that signals-of $(C) \sqsubseteq O \pi_{1}($ op $\downarrow C)$.

It is worth noting that Figure 7 does not include an analogue of TyComp-Subsume. This is deliberate because as we shall see below, process types reduce in conjunction with the processes they are assigned to, and the outcome is generally neither a sub- nor supertype of the original type.

\subsection{Type Safety}

We conclude the meta-theory of $\lambda_{æ}$ by proving type safety for its parallel part. Analogously to Section 3.4, we once again split type safety into separate proofs of progress and preservation.

4.4.1 Progress. We characterise the result forms of parallel processes by defining two judgements, ProcRes $\langle P\rangle$ and $\operatorname{ParRes}\langle P\rangle$, and by using the judgement RunRes $\langle\Psi \mid M\rangle$ from Section 3.4, as follows:

$$
\frac{\operatorname{ProcRes}\langle P\rangle}{\operatorname{ProcRes}\langle\uparrow \text { op }(V, P)\rangle} \quad \frac{\operatorname{ParRes}\langle P\rangle}{\operatorname{ProcRes}\langle P\rangle} \quad \frac{\operatorname{RunRes}\langle\emptyset \mid M\rangle}{\operatorname{ParRes}\langle\text { run } M\rangle} \quad \frac{\operatorname{ParRes}\langle P\rangle \quad \operatorname{ParRes}\langle Q\rangle}{\operatorname{ParRes}\langle P \| Q\rangle}
$$

These judgements express that a process $P$ is in a (top-level) result form $\operatorname{ProcRes}\langle P\rangle$ when, considered as a tree, it has a shape in which all signals are towards the root, parallel compositions are in the intermediate nodes, and individual computation results are at the leaves. Importantly, the computation results RunRes $\langle\emptyset \mid M\rangle$ we use here are those from which signals have been propagated out of (see Section 3.4.1). The finality of these results forms is then captured by the next lemma. 
Lemma 4.2. Given a process $P$ such that $\operatorname{ProcRes}\langle P\rangle$, then there exists no $Q$ such that $P \leadsto Q$.

We are now ready to state and prove the progress theorem for the parallel part of $\lambda_{\mathfrak{x}}$.

THEOREM 4.3. Given a well-typed closed process $\vdash P: C$, then either $(i)$ there exists a process $Q$ such that $P \leadsto Q$, or (ii) the process $P$ is already in a (top-level) result form, i.e., we have $\operatorname{ProcRes}\langle P\rangle$.

Proof. The proof is standard and proceeds by induction on the derivation of $\vdash P: C$. In the base case, when the derivation ends with the TyProc-Run rule, and $P=\operatorname{run} M$, we use Corollary 3.4.

4.4.2 Type Preservation. First, we note that the broadcast rules in Figure 6 introduce new inward propagating interrupts in their right-hand sides that originally do not exist in their left-hand sides. As a result, compared to the types one assigns to the left-hand sides of these reduction rules, the types assigned to their right-hand sides will need to feature corresponding type-level actions of these interrupts. We formalise this idea using a process type reduction relation $C \rightsquigarrow D$, given by

$$
\overline{X ! !(o, l) \rightsquigarrow X ! !(o, l)} \quad \overline{X ! ! \text { ops } \downarrow \downarrow(o, l) \rightsquigarrow X ! ! \text { ops } \downarrow \downarrow(\text { op } \downarrow(o, l))} \quad \frac{C \rightsquigarrow C^{\prime} \quad D \rightsquigarrow D^{\prime}}{C\left\|D \rightsquigarrow C^{\prime}\right\| D^{\prime}}
$$

where we write ops $\downarrow \downarrow(o, \iota)$ for a recursively defined action of a list of interrupts on $(o, \iota)$, given by

$$
[] \downarrow \downarrow(o, \iota) \stackrel{\text { def }}{=}(o, l) \quad(\text { op }:: \text { ops }) \downarrow \downarrow(o, l) \stackrel{\text { def }}{=} \text { op } \downarrow(\text { ops } \downarrow \downarrow(o, l))
$$

Intuitively, $C \rightsquigarrow D$ describes how process types reduce by being acted upon by freshly arriving interrupts. While we define the action behaviour only at the leaves of process types (under some enveloping sequence of actions), we can prove expected properties for arbitrary process types:

LEMMA 4.4.

(1) Process types can remain unreduced, i.e., $C \leadsto C$ for any process type $C$.

(2) Process types reduce by being acted upon, i.e., $C \rightsquigarrow$ op $\downarrow C$ for any type $C$ and interrupt op.

(3) Process types can reduce under enveloping actions, i.e., op $\downarrow C \rightsquigarrow$ op $\downarrow D$ when $C \rightsquigarrow D$.

(4) Process type reduction can introduce signals, i.e., signals-of $(C) \sqsubseteq_{O}$ signals-of $(D)$ when $C \rightsquigarrow D$.

For the proof of Lemma 4.4 (3), it is important that we introduce interrupts under an arbitrary enveloping sequence of interrupt actions, and not simply as $X ! !(o, \iota) \rightsquigarrow X ! !($ op $\downarrow(o, \iota))$. Further, the proof of Lemma 4.4 (4) requires us to generalise Lemma 3.1 (1) to lists of enveloping actions:

LEMMA 4.5. $\pi_{1}$ (ops $\left.\downarrow \downarrow(o, l)\right) \sqsubseteq O \pi_{1}$ (ops $\downarrow \downarrow($ op $\downarrow(o, l))$ )

As in Section 3.4.2, we again find it useful to define a separate typing judgement for evaluation contexts, this time written $\Gamma \vdash[C] \mathcal{F}: D$, together with an analogue of Lemma 3.5, which we omit here. Instead, we observe that this typing judgement is subject to process type reduction:

Lemma 4.6. Given $\Gamma \vdash[C] \mathcal{F}: D$ and $C \rightsquigarrow C^{\prime}$, then there exists $D^{\prime}$ with $D \rightsquigarrow D^{\prime}$ and $\Gamma \vdash\left[C^{\prime}\right] \mathcal{F}: D^{\prime}$.

We are now ready to state and prove the type preservation theorem for the parallel part of $\lambda_{æ}$.

Theorem 4.7. Given a well-typed process $\Gamma \vdash P: C$, such that $P$ can reduce as $P \leadsto Q$, then there exists a process type $D$, such that the process type $C$ can reduce as $C \rightsquigarrow D$, and we have $\Gamma \vdash Q: D$.

Proof. The proof proceeds by induction on the derivation of $P \leadsto Q$, using auxiliary typing inversion lemmas depending on the structure forced upon $P$ by the last rule used in $P \leadsto Q$. For all but the broadcast and evaluation context rules, we can pick $D$ to be $C$ and use Lemma 4.4 (1). For the broadcast rules, we define $D$ by introducing the corresponding interrupt, and build $C \rightsquigarrow D$ using the parallel composition rule together with Lemma 4.4 (2). For the evaluation context rule, we use Lemma 4.6 in combination with the induction hypothesis. Finally, in order to discharge effects-related side-conditions when commuting interrupts with signals, we use Lemma 4.1. 


\section{ASYNCHRONOUS EFFECTS IN ACTION}

We now show some examples of the kinds of programs one can write in $\lambda_{æ}$. Similarly to Section 2.6, we again allow ourselves access to mutable references, and use generic versions $\uparrow$ op $V$ of signals.

\subsection{Guarded Interrupt Handlers}

Before diving into the examples, we note that we often want the triggering of interrupt handlers to be based on not only the names of interrupts, but also the payloads that they carry. In order to express such more fine-grained triggering behaviour, we shall use a guarded interrupt handler:

promise (op $\mathrm{x}$ when guard $\mapsto$ comp) as $\mathrm{p}$ in cont

which is simply a syntactic sugar for the following interrupt handler that recursively reinstalls itself until the boolean guard becomes true, in which case it executes the handler code comp:

let rec waitForGuard ()$=$

promise (op $\mathrm{x} \mapsto$ if guard then comp else waitForGuard ()) as $\mathrm{p}^{\prime}$ in return $\mathrm{p}^{\prime}$ in

let $\mathrm{p}=$ waitForGuard () in cont

Here, $\mathrm{x}$ is bound both in guard and comp. Further, if comp has type $\langle X\rangle !\left(o^{\prime}, \iota^{\prime}\right)$ and cont has type $Y !(o, l)$, such that $\iota(\mathrm{op})=\left(o^{\prime}, \iota^{\prime}\right)$, then we can assign the entire computation the type $Y !\left(o, l \cup \iota_{h}\right)$, where the effect annotation $\iota_{h}$ is the least fixed point of the map $\iota^{\prime \prime} \mapsto\left\{\right.$ op $\left.\mapsto\left(o^{\prime}, \iota^{\prime} \cup \iota^{\prime \prime}\right)\right\}: I \rightarrow I$. Observe that some of the recursive encoding leaks into the type of the entire computation via $\iota_{h}$.

Note that regardless whether guard is true, every interrupt is propagated into cont. To typecheck their definition, and to ensure that guarded interrupt handlers are non-blocking, it is crucial that the handler code of ordinary interrupt handlers returns promise-typed values, as noted in Section 3.3.3.

\subsection{Preemptive Multi-Threading}

Multi-threading remains one of the most exciting applications of algebraic effects, with the possibility of expressing many evaluation strategies being the main reason for the extension of MULTICORE OCAML with effect handlers [Dolan et al. 2018]. These evaluation strategies are however cooperative in nature, where each thread needs to explicitly yield back control, stalling other threads until then.

While it is possible to also simulate preemptive multi-threading within the conventional treatment of algebraic effects, it requires a low-level access to the specific runtime environment, so as to inject yields into the currently running computation [Dolan et al. 2018]. In contrast, implementing preemptive multi-threading in $\lambda_{æ}$ is quite straightforward, and importantly, possible within the language itself-the injections into the running computation take the form of incoming interrupts.

For this, let us consider two interrupts, stop : 1 and go : 1, that communicate to a thread whether to pause or resume execution. These interrupts can originate from a timer process we run in parallel.

At the core of our implementation of preemptive multi-threading is the recursive function

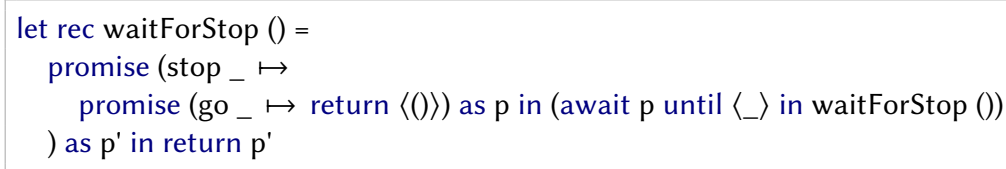

which first installs an interrupt handler for stop, letting subsequent computations run their course. Once the stop interrupt arrives, the interrupt handler for it is triggered and the next one for go gets installed. In contrast to the interrupt handler for stop, the one for go starts awaiting the (unit) promise $\mathrm{p}$. This means that any subsequent computations are blocked until a go interrupt is received, after which we recursively reinstall the interrupt handler for stop and repeat the cycle. 
To initiate the preemptive behaviour for some computation comp, we simply run the program waitForStop (); comp

The algebraicity reduction rules for interrupt handlers ensure that they propagate out of waitForStop and encompass the entire computation, including comp. Observe that in contrast to usual effect handler based encodings of multi-threading, waitForStop does not need any access to a thunk fun ()$\mapsto$ comp representing the threaded computation. In particular, the given computation comp can be completely unaware of the multi-threaded behaviour, both in its definition and its type.

This approach can be easily extended to multiple threads, by using interrupts' payloads to communicate thread IDs. To this end, we can consider interrupts stop : int and go : int, and define

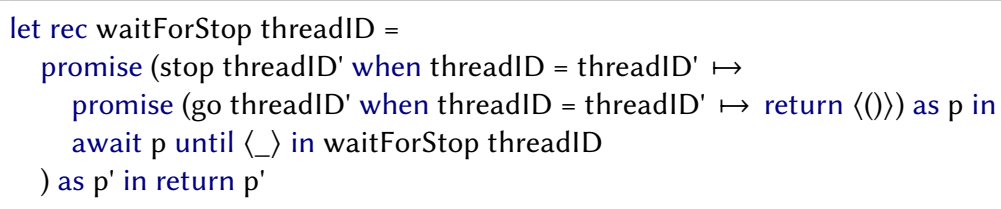

using guarded interrupt handlers, and conditioning their triggering based on the received IDs.

\subsection{Remote Function Calls}

One of the main uses of asynchronous computation is to offload the execution of long-running functions $f: A \rightarrow B !(o, \iota)$ to remote processes. Below we show how to implement this in $\lambda_{æ}$.

One invokes a remote function by issuing a signal named call with the function's argument, and then awaits an interrupt named result with the function's result, with all effects specified by $(o, \iota)$ happening at the callee site. The caller then calls such a remote function through a wrapper callWith, which issues the call signal, installs a handler for the result interrupt, and returns a thunk that awaits the function's result. For instance, one may then use remote functions in their code as

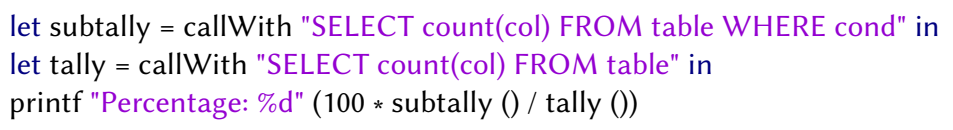

To avoid the results of earlier remote function calls from fulfilling the promises of later ones, we assign to each call a unique identifier, and communicate those in payloads. We implement these unique identifiers using a counter. For a remote function $f: A \rightarrow B !(o, l)$, we type the signals and interrupts as call : $A \times$ int and result : $B \times$ int. The caller site function callWith is then defined as

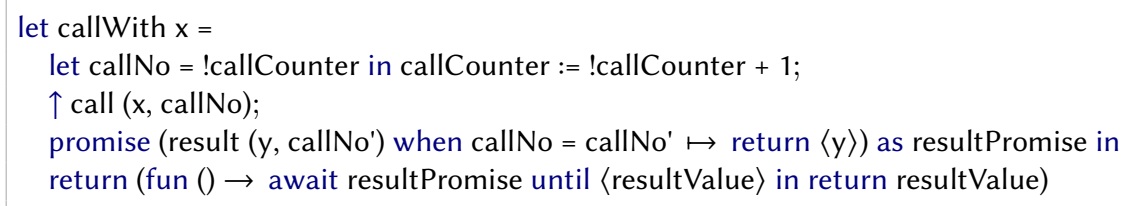

After issuing the call signal, callWith installs a guarded interrupt handler for the corresponding result interrupt, and then returns a function that, when called, awaits the result of the remote call.

At the callee site, we simply install an interrupt handler that executes the function in question, issues an outgoing signal with the function's result, and then recursively reinstalls itself, as follows:

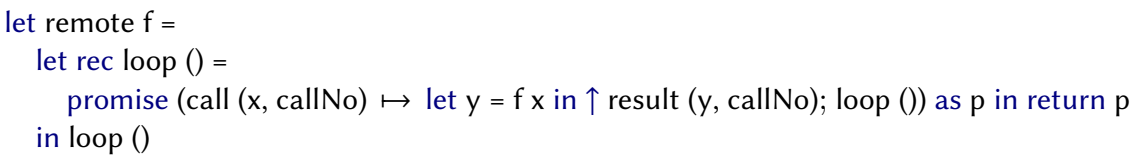


Unlike effect handlers, our interrupt handlers have very limited control over the execution of their continuation. However, we can still simulate cancellations of asynchronous computations by awaiting a promise that will never be fulfilled. We achieve this with the help of the function

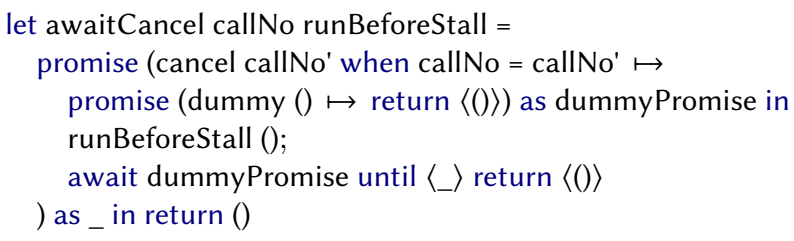

which takes the identifier of the remote function call that we want to make cancellable, and a thunked computation to run before the continuation is stalled. We can then extend the callee site with cancellable function calls by invoking awaitCancel before we start executing the long-running computation $\mathrm{f} x$. In particular, we change the interrupt handler code in remote $f$ to read as follows:

call $(\mathrm{x}$, callNo) $\mapsto$ awaitCancel callNo loop; let $\mathrm{y}=\mathrm{f} \mathrm{x}$ in $\uparrow$ result ( $\mathrm{y}$, callNo); loop ()

However, if left as is, cancelling one call would cancel all unfinished remote function calls because they would be part of the stalled continuation. To overcome this, we run the callee site in parallel with an auxiliary process (which we omit here) that reacts to a cancel interrupt by reinvoking these unfinished calls (minus the cancelled one) by reissuing the corresponding call signals, which then get propagated to the callee site, and to the loop () we run in awaitCancel callNo loop before stalling.

We note that the cancelled computation is only perpetually stalled, but not discarded completely, leading to a memory leak. We conjecture that extending $\lambda_{æ}$ with effect handlers that have greater control over the continuation could lead to a more efficient code for the callee site. We also conjecture that a future extension of $\lambda_{x}$ with dynamic process creation would eliminate the need for the auxiliary reinvoker process, because then the callee site could create a new process for every remote function call it receives, and each cancel interrupt would stall only one of such (sub-)processes.

\subsection{Runners of Algebraic Effects}

Next, we use $\lambda_{æ}$ to implement a parallel variant of runners of algebraic effects [Ahman and Bauer 2020]. These are a natural mathematical model and programming abstraction for resource management based on algebraic effects, and correspond to effect handlers that apply continuations (at most) once in a tail call position. In a nutshell, for a signature of operation symbols op : $A_{\mathrm{op}} \rightarrow B_{\mathrm{op}}$, a runner $\mathcal{R}$ comprises a family of stateful functions $\overline{\mathrm{op}}_{\mathcal{R}}: A_{\mathrm{op}} \rightarrow R \Rightarrow B_{\mathrm{op}} \times R$, called co-operations, where $R$ is the type of resources that the runner manipulates. In the more general setting of Ahman and Bauer [2020], the co-operations also model other, external effects, such as native calls to the operating system, and can furthermore raise exceptions-all of which we shall gloss over here.

Given a runner $\mathcal{R}$, Ahman and Bauer [2020] provide the programmer with a construct

$$
\text { using } \mathcal{R} @ V_{\text {init }} \text { run } M \text { finally }\left\{\text { return } x @ r_{\text {fin }} \mapsto N\right\}
$$

which runs $M$ using $\mathcal{R}$, with resources initially set to $V_{\text {init }}$; and finalises the return value and final resources using $N$, e.g., ensuring that all file handles get closed. This is a form of effect handling: it executes $M$ by invoking co-operations in place of operation calls, while doing resource-passing under the hood. Below we show by means of examples how one can use $\lambda_{æ}$ to naturally separate $\mathcal{R}$ and $M$ into different processes. For simplicity, we omit the initialisation and finalisation phases.

For our first example, let us consider a runner that implements a pseudo-random number generator by providing a co-operation for random : $1 \rightarrow$ int, which we can for example implement as

let linearCongruenceGeneratorRunner modulus a $\mathrm{c}$ initialSeed = 


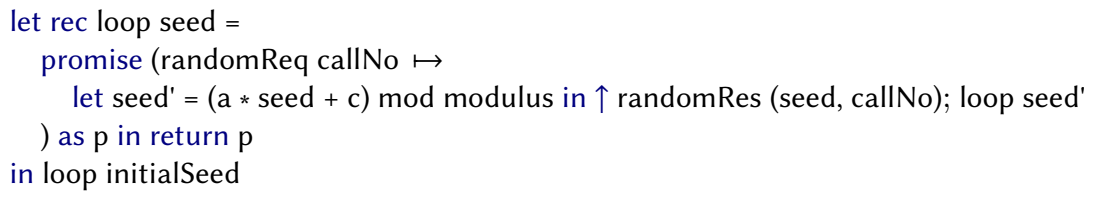

It is given by a recursive interrupt handler, which listens for randomReq : int requests issued by clients, and itself issues randomRes : int $\times$ int responses. The resource this runner manages is the seed, which it passes between subsequent co-operation calls as an argument to the recursive loop.

For the client code $M$, we implement operation calls random () as discussed in Section 2.2, by decoupling them into signals and interrupts. We again use guarded interrupt handlers and call identifiers to avoid a response to one operation call fulfilling the promises of subsequent ones.

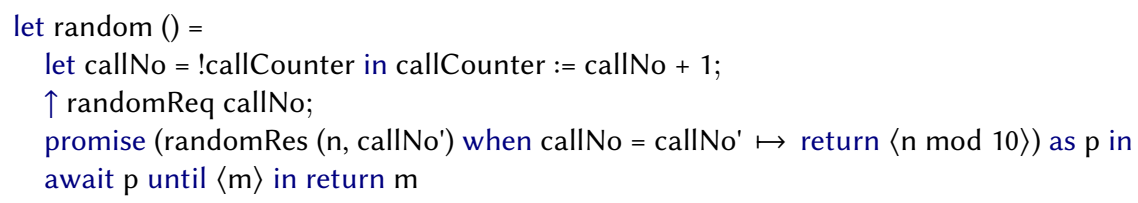

As a second example, we show that this parallel approach to runners naturally extends to multiple co-operations. Specifically, we implement a runner for a heap, by providing co-operations for

$$
\text { alloc : int } \rightarrow \text { loc lookup : loc } \rightarrow \text { int } \quad \text { update }: \text { loc } \times \text { int } \rightarrow 1
$$

We represent these co-operations using a signal/interrupt pair (opReq, opRes) with payload types

type payloadReq $=\mid$ AllocReq of int $\mid$ LookupReq of loc $\mid$ UpdateReq of loc $*$ int

type payloadRes $=\mid$ AllocRes of loc $\mid$ LookupRes of int $\mid$ UpdateRes of unit

The resulting runner is then implemented by pattern-matching on the payload value as follows:

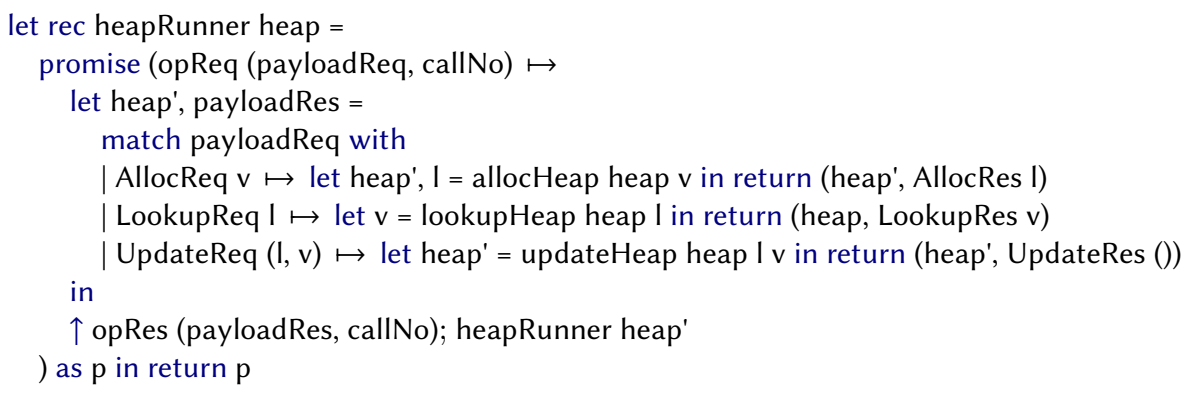

Note that by storing heap in memory, we could have also used three signal/interrupt pairs and split heapRunner into three distinct interrupt handlers, one for each of allocation, lookup, and update.

\subsection{Non-Blocking Post-Processing of Promised Values}

As discussed in Section 2.4, interrupt handlers differ from ordinary operation calls by allowing user-side post-processing of received data. In this final example, we show that $\lambda_{æ}$ is flexible enough to modularly perform further non-blocking post-processing of this data anywhere in a program.

For instance, let us assume we are writing a program that contains an interrupt handler (for some op) that promises to return us a list of integers. Now, at some later point in the program, we decide that we want to further process this list if and when it becomes available, e.g., by using some of its elements to issue an outgoing signal. Of course, we could do this by going back and changing 
the original interrupt handler, but this would not be very modular; nor do we want to block the entire program's execution (using await) until op arrives and the concrete list becomes available.

Instead, we can define a generic combinator for non-blocking post-processing of promised values process $_{\mathrm{op}} \mathrm{p}$ with $(\langle\mathbf{x}\rangle \mapsto \mathrm{comp})$ as $\mathrm{q}$ in cont

that takes an earlier made promise $p$ (which we assume originates from handling the specified interrupt op), and makes a new promise to execute the post-processing code comp[v/x] once $p$ gets fulfilled with some value v. The (non-blocking) continuation cont can refer to comp's result using the new promise-typed variable $q$ bound in it. Under the hood, process ${ }_{\mathrm{op}}$ is a syntactic sugar for

promise (op _ $\mapsto$ await $\mathrm{p}$ until $\langle\mathrm{x}\rangle$ in let $\mathrm{y}=$ comp in return $\langle\mathrm{y}\rangle$ ) as $\mathrm{q}$ in cont

While process op does involve an await, it gets exposed only after op is received, but by that time $p$ will have been fulfilled with some $v$ by an earlier interrupt handler, and thus the await can reduce.

Returning to post-processing a list of integers promised by an existing interrupt handler, below is an example showing the use of the process op combinator and how to chain multiple post-processing computations together (here, filtering, folding, and issuing an outgoing signal), in the same spirit as how one is taught to program compositionally with futures and promises [Haller et al. 2020]:

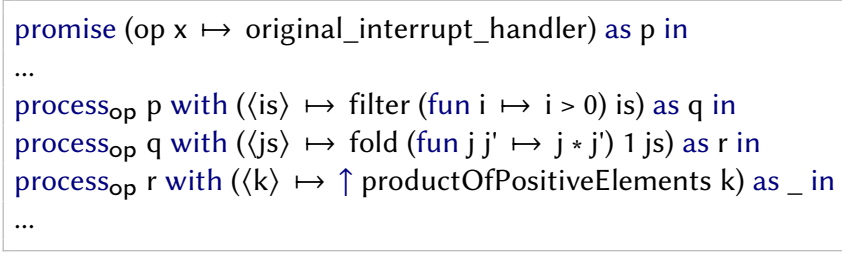

We note that for this to work, it is crucial that incoming interrupts behave like (deep) effect handling (see Section 3.2) so that all three post-processing computations get executed, in their program order.

\section{CONCLUSION}

We have shown how to incorporate asynchrony within algebraic effects, by decoupling the execution of operation calls into signalling that an operation's implementation needs to be executed, and interrupting a running computation with the operation's result, to which it can react by installing interrupt handlers. We have shown that our approach is flexible enough that not all signals have to have a matching interrupt, and vice versa, allowing us to also model spontaneous behaviour, such as a user clicking a button or the environment preempting a thread. We have formalised these ideas in a small calculus, called $\lambda_{æ}$, and demonstrated its flexibility on a number of examples. We have also accompanied the paper with an AGDA formalisation and a prototype implementation of $\lambda_{æ}$. However, various future work directions still remain. We discuss these and related work below.

Asynchronous effects. As asynchrony is desired in practice, it is no surprise that KoKA [Leijen 2017] and Multicone OCAML [Dolan et al. 2018], the two largest implementations of algebraic effects and handlers, have been extended accordingly. In KoKA, algebraic operations reify their continuation into an explicit callback structure that is then dispatched to a primitive such as setTimeout in its Node.JS backend. In Multicore OCAML, one uses low-level functions such as set_signal or timer_create that modify the runtime by interjecting operation calls inside the currently running code. Both approaches thus delegate the actual asynchrony to existing concepts in their backends. In contrast, in $\lambda_{x}$, we can express such backend features within the core calculus itself.

Further, in $\lambda_{\circledast}$, we avoid having to manually use (un)masking to disable asynchronous effects in unwanted places in our programs, which can be a very tricky business to get right, as noted by Dolan et al. [2018]. Instead, by design, interrupts in $\lambda_{æ}$ never influence running code unless the 
code has an explicit interrupt handler installed, and they always wait for any potential handler to present itself during execution (recall that they get discarded only when reaching a return).

Message-passing. While in this paper we have focussed on the foundations of asynchrony in the context of algebraic effects, the ideas we propose have also many common traits with concurrency models based on message-passing, such as the Actor model [Hewitt et al. 1973], the $\pi$-calculus [Milner et al. 1992], and the join-calculus [Fournet and Gonthier 1996], just to name a few. Namely, one can view the issuing of a signal $\uparrow$ op $(V, M)$ as sending a message, and handling an interrupt $\downarrow$ op $(W, M)$ as receiving a message, along a channel named op. In fact, we believe that in our prototype implementation we could replace the semantics presented in the paper with an equivalent one based on shared channels (one for each op), to which the interrupt handlers could subscribe to. Instead of propagating signals first out and then in, they would be sent directly to channels where interrupt handlers immediately receive them, drastically reducing the cost of communication.

Comparing $\lambda_{\mathfrak{x}}$ to the Actor model, we see that the run $M$ processes evolve in their own bubbles, and only communicate with other processes via signals and interrupts, similarly to actors. However, in contrast to messages not being required to be ordered in the Actor model, in our $P \| Q$, the process $Q$ receives interrupts in the same order as the respective signals are issued by $P$ (and vice versa). This communication ordering could be relaxed by allowing signals to be hoisted out of computations from deeper than just the top level. Another difference with actors is that $\lambda_{x^{-}}$-computations can react to interrupts only sequentially, and not by dynamically creating new parallel processes-first-class parallel processes and their dynamic creation is something we plan to address in future work.

It is worth noting that our interrupt handlers are similar to the message receiving construct in the $\pi$-calculus, in that they both synchronise with matching incoming interrupts/messages. However, the two are also different, in that interrupt handlers allow reductions to take place under them and non-matching interrupts to propagate past them. Further, our interrupt handlers are also similar to join definitions in the join-calculus, describing how to react when a corresponding interrupt arrives or join pattern appears, where in both cases the reaction could involve effectful code. To this end, our interrupt handlers resemble join definitions with simple one-channel join patterns. However, where the two constructs differ is that join definitions also serve to define new (local) channels, similarly to the restriction operator in the $\pi$-calculus, whereas we assume a fixed global set of channels (i.e., signal and interrupt names). We expect that extending $\lambda_{æ}$ with local algebraic effects [Biernacki et al. 2019; Staton 2013] could help us fill this gap between the formalisms.

Scoped operations. As discussed in Section 3.2, despite their name, interrupt handlers behave like algebraic operations, not like effect handlers. However, one should also note that they are not conventional operations because they carry computational data that sequential composition does not interact with, and that only gets triggered when a corresponding interrupt is received.

Such generalised operations are known in the literature as scoped operations [Piróg et al. 2018], a leading example of which is spawn $(M ; N)$, where $M$ is the new child process to be executed and $N$ is the current process. Crucially, the child $M$ should not directly interact with the current process. Scoped operations achieve this behaviour by declaring $M$ to be in the scope of spawn, resulting in let $x=\operatorname{spawn}(M ; N)$ in $K \leadsto \operatorname{spawn}(M$; let $x=N$ in $K)$, exactly as we have for interrupt handlers.

Further recalling Section 3.2, despite their appearance, incoming interrupts behave computationally like effect handling, not like algebraic operations. In fact, it turns out they correspond to effect handling induced by an instance of scoped effect handlers [Piróg et al. 2018]. Compared to ordinary effect handlers, scoped effect handlers explain both how to interpret operations and their scopes. In our setting, this corresponds to selectively executing the handler code of interrupt handlers.

It would be interesting to extend our work both with scoped operations having more general signatures, and with additional effect handlers for them. The latter could allow preventing the 
propagation of incoming interrupts into continuations, discarding the continuation of a cancelled remote call, and techniques such as masking or reordering interrupts according to priority levels.

Modal types. We recall that the type safety of $\lambda_{æ}$ crucially relies on the promise-typed variables bound by interrupt handlers not being allowed to appear in the payloads of signals. This ensures that it is safe to propagate signals past all enveloping interrupt handlers, and communicate their payloads to other processes. In its essence, this is similar to the use of modal types in distributed [Murphy VII 2008] and reactive programming [Bahr et al. 2019; Krishnaswami 2013] to classify values that can travel through space and time. In our case, it is the omission of promise types from ground types that allows us to consider the payloads of signals and interrupts as such mobile values

We expect that these connections to modal types will be key for extending $\lambda_{æ}$ with (i) higher-order payloads and (ii) process creation. For (i), we want to avoid the bodies of function-typed payloads to be able to await enveloping promise variables to be fulfilled. For (ii), we want to do the same for the dynamically created processes. In both cases, the reason is to be able to safely propagate the corresponding programming constructs past enveloping interrupt handlers, and eventually hoist them out of individual computations. We believe that the more structured treatment of contexts $\Gamma$, as studied in various modal type systems, will hold the key for these extensions to be type safe.

Denotational semantics. In this paper we study only the operational side of $\lambda_{\mathfrak{x}}$, and leave developing its denotational semantics for the future. In light of how we have motivated the $\lambda_{x}$-specific programming constructs, and based on the above discussions, we expect the denotational semantics to take the form of an algebraically natural monadic semantics, where the monad would be given by an instance of the one studied by Piróg et al. [2018] for scoped operations (quotiented by the commutativity of signals and interrupt handlers, and extended with nondeterminism to model different evaluation outcomes), incoming interrupts would be modelled as homomorphisms induced by scoped algebras, and parallel composition by considering all nondeterministic interleavings of (the outgoing signals of) individual computations, e.g., based on how Plotkin [2012] and Lindley et al. [2017] model it in the context of general effect handlers. Finally, we expect to take inspiration for the denotational semantics of the promise type from that of modal logics and modal types.

Reasoning about asynchronous effects. In addition to using $\lambda_{x}$ 's type-and-effect system only for specification purposes (such as specifying that $M: X !(\emptyset,\{\})$ raises no signals and installs no interrupt handlers), we wish to make further use of it for validating effect-dependent optimisations [Kammar and Plotkin 2012]. For instance, whenever $M: X !(o, \iota)$ and $\iota($ op $)=\perp$, we would like to know that $\downarrow$ op $(V, M) \sim^{*} M$. One way to validate such optimisations is to develop an adequate denotational semantics, and then use a semantic computational induction principle [Bauer and Pretnar 2014; Plotkin and Pretnar 2008]. For $\lambda_{æ}$, this would amount to only having to prove the optimisations for return values, signals, and interrupt handlers. Another way to validate effectdependent optimisations would be to define a suitable logical relation for $\lambda_{æ}$ [Benton et al. 2014].

In addition to optimisations based on $\lambda_{æ}$ 's existing effect system, we plan to explore extending processes and their types with communication protocols inspired by session types [Honda et al. 1998], so as to refine the current "broadcast everything everywhere" communication strategy.

\section{ACKNOWLEDGEMENTS}

We thank the anonymous reviewers, Otterlo IFIP WG 2.1 meeting participants, and Andrej Bauer, Gavin Bierman, Žiga Lukšič, and Alex Simpson for their useful feedback. This project has received funding from the European Union's Horizon 2020 research and innovation programme under the Marie Skłodowska-Curie grant agreement No 834146 . This material is based upon work supported by the Air Force Office of Scientific Research under award number FA9550-17-1-0326. 


\section{REFERENCES}

D. Ahman. 2020. AgDA formalisation of the $\lambda_{\mathfrak{x}}$-calculus. Available at https://github.com/danelahman/aeff-agda/releases/ tag/popl-2021.

D. Ahman and A. Bauer. 2020. Runners in action. In Proc. of 29th European Symp. on Programming, ESOP 2020 (LNCS, Vol. 12075). Springer, 29-55.

D. Ahman and M. Pretnar. 2020. Software artefact for the POPL 2021 paper "Asynchronous Effects". Available at https: //doi.org/10.5281/zenodo.4072753.

R. M. Amadio and P-L. Curien. 1998. Domains and Lambda Calculi. Cambridge University Press.

P. Bahr, C. Graulund, and R. E. Mogelberg. 2019. Simply RaTT: a fitch-style modal calculus for reactive programming without space leaks. Proc. ACM Program. Lang. 3, ICFP (2019), 109:1-109:27.

A. Bauer and M. Pretnar. 2014. An Effect System for Algebraic Effects and Handlers. Logical Methods in Computer Science 10, 4 (2014).

A. Bauer and M. Pretnar. 2015. Programming with algebraic effects and handlers. f. Log. Algebr. Meth. Program. 84, 1 (2015), 108-123.

N. Benton, M. Hofmann, and V. Nigam. 2014. Abstract effects and proof-relevant logical relations. In Proc. of 41st Ann. ACM SIGPLAN-SIGACT Symp. on Principles of Programming Languages, POPL 2014. ACM, 619-632.

D. Biernacki, M. Piróg, P. Polesiuk, and F. Sieczkowski. 2019. Abstracting Algebraic Effects. Proc. ACM Program. Lang. 3, POPL (2019), 6:1-6:28.

E. Bingham, J. P. Chen, M. Jankowiak, F. Obermeyer, N. Pradhan, T. Karaletsos, R. Singh, P. Szerlip, P. Horsfall, and N. D. Goodman. 2019. Pyro: Deep Universal Probabilistic Programming. F. Mach. Learn. Res. 20, 1 (Jan. 2019), 973-978.

L. Convent, S. Lindley, C. McBride, and C. McLaughlin. 2020. Doo bee doo bee doo. f. Funct. Program. 30 (2020), e9.

S. Dolan, S. Eliopoulos, D. Hillerström, A. Madhavapeddy, K. C. Sivaramakrishnan, and L. White. 2018. Concurrent System Programming with Effect Handlers. In Proc. of 18th Int. Sym. Trends in Functional Programming, TFP 2017. Springer, 98-117.

C. Fournet and G. Gonthier. 1996. The Reflexive CHAM and the Join-Calculus. In Proc. of 23rd ACM SIGPLAN-SIGACT Symp. on Principles of Programming Languages, POPL'96. ACM, 372-385.

G. Gierz, K. H. Hofmann, K. Keimel, J. D. Lawson, M. Mislove, and D. S. Scott. 2003. Continuous Lattices and Domains. Number 93 in Encyclopedia of Mathematics and its Applications. Cambridge University Press.

P. Haller, A. Prokopec, H. Miller, V. Klang, R. Kuhn, and V. Jovanovic. 2020. Scala documentation: Futures and Promises. (July 2020). Available online at https://docs.scala-lang.org/overviews/core/futures.html.

C. Hewitt, P. Bishop, and R. Steiger. 1973. A Universal Modular ACTOR Formalism for Artificial Intelligence. In Proc. of 3rd Int. foint Conf. on Artificial Intelligence, IfCAI'73. Morgan Kaufmann Publishers Inc., 235-245.

K. Honda, V. T. Vasconcelos, and M. Kubo. 1998. Language Primitives and Type Discipline for Structured CommunicationBased Programming. In Proc. of 7th European Symp. on Programming, ESOP 1998 (LNCS, Vol. 1381). Springer, 122-138.

O. Kammar, S. Lindley, and N. Oury. 2013. Handlers in Action. In Proc. of 18th ACM SIGPLAN Int. Conf. on Functional Programming, ICFP 2013. ACM, 145-158.

O. Kammar and G. D. Plotkin. 2012. Algebraic foundations for effect-dependent optimisations. In Proc. of 39th ACM SIGPLAN-SIGACT Symp. on Principles of Programming Languages, POPL 2012. ACM, 349-360.

N. R. Krishnaswami. 2013. Higher-Order Functional Reactive Programming without Spacetime Leaks. In Proc of 18th ACM SIGPLAN Int. Conf. on Functional Programming, ICFP 2013. ACM, 221-232.

D. Leijen. 2017. Structured asynchrony with algebraic effects. In Proc. of 2nd ACM SIGPLAN Int. Wksh. on Type-Driven Development, TyDe@ICFP 2017. ACM, 16-29.

P. B. Levy, J. Power, and H. Thielecke. 2003. Modelling environments in call-by-value programming languages. Inf. Comput. 185, 2 (2003), 182-210.

S. Lindley, C. McBride, and C. McLaughlin. 2017. Do Be Do Be Do. In Proc. of 44th ACM SIGPLAN Symp. on Principles of Programming Languages, POPL 2017. ACM, 500-514.

R. Milner, J. Parrow, and D. Walker. 1992. A calculus of mobile processes, I. Inf. Comput. 100, 1 (1992), 1-40.

T. Murphy VII. 2008. Modal Types for Mobile Code. Ph.D. Dissertation. Carnegie Mellon University.

M. Piróg, T. Schrijvers, N. Wu, and M. Jaskelioff. 2018. Syntax and Semantics for Operations with Scopes. In Proc. of 33rd Annual ACM/IEEE Symp. on Logic in Computer Science, LICS 2018. ACM, 809-818.

G. D. Plotkin. 2012. Concurrency and the algebraic theory of effects. (2012). Invited talk at the 23rd Int. Conf. on Concurrency Theory, CONCUR 2012.

G. D. Plotkin and J. Power. 2002. Notions of Computation Determine Monads. In Proc. of 5th Int. Conf. on Foundations of Software Science and Computation Structures, FOSSACS 2002 (LNCS, Vol. 2303). Springer, 342-356.

G. D. Plotkin and M. Pretnar. 2008. A Logic for Algebraic Effects. In Proc. of 23th Ann. IEEE Symp. on Logic in Computer Science, LICS 2008. IEEE, 118-129.

G. D. Plotkin and M. Pretnar. 2013. Handling Algebraic Effects. Logical Methods in Computer Science 9, 4:23 (2013). 
L. Poulson. 2020. Asynchronous Effect Handling. Master's thesis. School of Informatics, University of Edinburgh.

M. Pretnar. 2015. An Introduction to Algebraic Effects and Handlers. Invited tutorial paper. Electr. Notes Theor. Comput. Sci. 319 (2015), 19-35.

M. Pretnar. 2020. Programming language ÆFF. Available at https://github.com/matijapretnar/aeff/releases/tag/popl-2021.

J. Schwinghammer. 2002. A Concurrent Lambda-Calculus with Promises and Futures. Master's thesis. Programming Systems Lab, Universität des Saarlandes.

S. Staton. 2013. Instances of Computational Effects: An Algebraic Perspective. In Proc. of 28th Ann. ACM/IEEE Symp. on Logic in Computer Science, LICS 2013. IEEE, 519-519.

S. Staton. 2015. Algebraic Effects, Linearity, and Quantum Programming Languages. In Proc. of 42nd Annual ACM SIGPLANSIGACT Symp. on Principles of Programming Languages, POPL 2015. ACM, 395-406.

A. K. Wright and M. Felleisen. 1994. A Syntactic Approach to Type Soundness. Information and Computation 115, 1 (1994), $38-94$. 